to describe the experimental results. This in not unexpected since, in three of the elements studied (Al, Si, and $\mathrm{Fe}$ ), there is a resonance in the total cross section of a width comparable to the energy resolution of the present experiment.

(2) For each element, the best fit to both the scattering and the polarization was obtained with the same set of parameters. This is shown most strikingly in case of $\mathrm{Al}^{27}$, where the opposite sign of the polarization, and the best fit to the elastic scattering, were both obtained with the same large change in $V_{\mathrm{CR}}$. The analysis shows that there is a strong correlation between the fluctuations away from the optical model in the elastic scattering and in the associated polarization.

(3) The addition of compound elastic scattering improved the agreement between calculation and experiment for both scattering and polarization in all the cases studied, and for some of the curves, was essential to give any semblance of an optical-model fit.

\section{ACKNOWLEDGMENTS}

The author would like to thank Dr. H. E. Wegner for considerable advice and encouragement, and Dr. G. N. Glasoe for several useful discussions. Thanks are also due to I. Feigenbaum and K. Rosenbaum for a great deal of help and cooperation during the operation of the experiment. Finally, the author would like to thank E. Auerbach, who made his ABACUs-2 code available and carried out the computer calculations described in this paper.

\title{
Inelastic $\pi^{-}-p$ Interactions in the Energy Region of 310 to $454 \mathrm{MeV}^{*}$
}

\author{
Barry C. Barish, $\dagger$ Richard J. Kurz, Victor Perez-Mendez, and Julius Solomonł \\ Lawrence Radiation Laboratory, University of California, Berkeley, California
}

(Received 11 March 1964)

\begin{abstract}
Differential cross sections for positive pions, protons, and neutrons resulting from inelastic $\pi^{-}-p$ collisions have been measured from 310- to 454-MeV incident-pion kinetic energy. The data were obtained with electronic counter systems, which measured the energy distribution of the final-state particle of interest at a series of fixed angles. The results have been interpreted in terms of the final states $\pi^{+} \pi^{-} n, \pi^{0} \pi^{0} n$, and $\pi^{-} \pi^{0} p$. The total cross sections for these three modes as a function of incident pion energy are in qualitative agreement with the predictions by Schnitzer. A preference is shown for his set of $\pi-\pi$ scattering lengths; $a_{0}=0.65$, $a_{1}=0.07$, and $a_{2}=-0.14 \mu^{-1}$. The observed neutron distributions correspond to a strong preference for low c.m.system neutron energies in both the $\pi^{+} \pi^{-} n$ and $\pi^{0} \pi^{0} n$ final states. The effect is not present in the observed proton distributions from the $\pi^{-} \pi^{0} p$ reaction, which suggests that it is due to a $I=0, \pi-\pi$ interaction. The $\pi^{+}$data show the formation of the $(3,3)$ isobar combination of the $\pi^{-}-n$ system in the $\pi^{+} \pi^{-} n$ final state. Analysis in terms of an isobar model indicates the predominance of $I=1 / 2$ incident state.
\end{abstract}

\section{INTRODUCTION}

W $\mathrm{E}$ have performed a series of measurements to investigate the inelastic channels available to the $\pi^{-}-p$ system in the region between $310-$ and $454-\mathrm{MeV}$ incident $\pi^{-}$kinetic energy. In the analysis of these measurements we have assumed that the single-pionproduction channels listed below are the dominant inelastic reactions:

$$
\begin{array}{cc}
\pi^{-}+p \rightarrow \pi^{+}+\pi^{-}+n & \left(\pi^{+} \pi^{-} n\right) \\
\pi^{-}+p \rightarrow \pi^{-}+\pi^{0}+p & \left(\pi^{-} \pi^{0} p\right) \\
\pi^{-}+p \rightarrow \pi^{0}+\pi^{0}+n & \left(\pi^{0} \pi^{0} n\right) .
\end{array}
$$

Double-pion production has been neglected.

Three separate experiments were performed with an

* Work done under the auspices of the U. S. Atomic Energy Commission.

$\dagger$ Present address: Synchrotron Laboratory, California Institute of Technology, Pasadena, California.

‡Present address: Palmer Physical Laboratory, Princeton University, Princeton, New Jersey. internal target of the Berkeley 184-in. synchrocyclotron as the source of pions. A magnetic beam-transport system momentum-analyzed and focused the $\pi^{-}$beam at a liquid-hydrogen target. In each experiment one of the three final-state particles was detected by an electroniccounter system. In the first experiment the final-state particle detected was a $\pi^{+}$, which is produced only in $\pi^{+} \pi^{-} n$. The following two experiments were concerned with the proton from $\pi^{-} \pi^{0} p$ and the neutrons from $\pi^{+} \pi^{-} n$ and $\pi^{0} \pi^{0} n$.

The emphasis of this paper is upon the results of the measurements. Consequently a description of the experimental methods and data analysis is deferred to the end of the paper (Sec. III). The reader is referred to Ref. 1 for detailed discussion of any aspects of the work reported here. In Sec. II the results of the three meas-

\footnotetext{
${ }^{1}$ Barry C. Barish, Ph.D. thesis, Lawrence Radiation Laboratory Report UCRL-10470, August 1962 (unpublished); Richard J. Kurz, Ph.D. thesis, Lawrence Radiation Laboratory Report UCRL-10564, December 1962 (unpublished); Julius Solomon, Ph.D. thesis, Lawrence Radiation Laboratory Report UCRL10585, January 1963 (unpublished).
} 
urements are presented; the final portion of Sec. II gives a discussion of the results in terms of the theoretical models and other experimental data.

To provide background before presenting the results, a short resumé of the theoretical work on the singlepion-production reactions follows.

After the success of the static model in explaining the $(3,3)$ resonance in the pion-nucleon system, several attempts were made to extend it to single-pion production at low energies. ${ }^{2}$ Measurements of the total cross section for $\pi^{+} \pi^{-} n$ from threshold to $430-\mathrm{MeV}$ incident- $\pi^{-}$ energy proved to be an order of magnitude larger than these static-model predictions. ${ }^{3}$ Theoretical attempts were then made to extend the static model by including a $\pi-\pi$ interaction and/or a $(3,3)$ isobar final-state interaction. This approach of decomposing the threebody problem into several two-body problems has been the most frequently used to date.

Rodberg proposed that the size of the cross section might be due to an interaction of the incident $\pi$ and the $\pi$ cloud of the nucleon. He considered $S$ - and $P$-wave $\pi-\pi$ interactions in a zero-range approximation and showed that the rapid increase of the total cross section for $\pi^{+} \pi^{-} n$ as a function of incident energy could be accounted for with a model of this type. ${ }^{4}$

Another approach to explain the data of Perkins et al. was proposed by Anisovich. ${ }^{5} \mathrm{He}$ assumes that the energy dependence of the matrix element is due only to the $(3,3)$ resonance interaction between the nucleon and one of the mesons in the final state. Anisovich characterizes the reaction in both the $I=\frac{1}{2}$ and $I=\frac{3}{2}$ states by three parameters, one for $S$-wave and two for $P$-wave production of the $(3,3)$ isobar combination. He by-passes the actual pion-energy spectra by performing an integration over energy and derives expressions for total cross sections and for the angular distributions of the pions in terms of the six parameters of his model. The results of the model for the total cross section of $\pi^{+} \pi^{-} n$ and the $\pi^{+}$angular distributions are in agreement with the data of Perkins et al., but the available experimental data were not sufficient to provide a decisive test of the validity of the model because of the large number of unknown parameters.

A detailed analysis of single $\pi$ production, including $\pi-\pi$ interaction and $\pi-N$ final-state interactions, was made by Goebel and Schnitzer. ${ }^{6}$ Again by adding $S$ and $P$-wave $\pi-\pi$ terms to the static-model formalism, Goebel and Schnitzer calculated total cross sections and

\footnotetext{
2 Saul Barshay, Phys. Rev. 103, 1102 (1956); Jerrold Franklin, Phys. Rev. 105, 1101 (1957) ; Leonard S. Rodberg, Phys. Rev. 106, 1090 (1957); Emil Kazes, Phys. Rev. 107, 1131 (1957).

${ }^{3}$ Walton A. Perkins, III, John C. Caris, Robert W. Kenney, and Victor Perez-Mendez, Phys. Rev. 118, 1364 (1960).

${ }^{4}$ Leonard S. Rodberg, Phys. Rev. Letters 3, 58 (1959).

${ }^{5}$ V. V. Anisovich, Zh. Eksperim. i Teor. Fiz. 39, 97 (1960) [English transl.: Soviet Phys.-JETP 12, 71 (1961)]; V. V. Anisovich, Zh. Eksperim. i Teor. Fiz. 39, 1357 (1960) [English transl. : Soviet Phys.-JETP 12, 946 (1961)].

${ }^{6}$ Charles J. Goebel and Howard J. Schnitzer, Phys. Rev. 123, 1021 (1961).
}

pion angular distributions in terms of $S$ - and $P$-wave coupling constants $\lambda_{s}$ and $\lambda_{p}$, which are related to $a_{0}$, $a_{1}$, and $a_{2}$, where $a_{I}$ is the scattering length for the $\pi-\pi$ isotopic-spin state $I ; a_{0}$ was assumed to be $5 a_{2} / 2$. No quantitative predictions can be made by their model concerning the pion-energy distributions because of their approximate treatment of phase space. They chose the value of their parameters to fit the total cross section for $\pi^{+} \pi^{-} n$ as a function of energy up to $430 \mathrm{MeV}$. They predicted the $\pi^{+}$angular distribution to have a strong forward and backward peaking in the c.m. system. The data of Perkins et al. ${ }^{3}$ and preliminary results of one of the measurements reported here both showed much more isotropy in the $\pi^{+}$angular distribution than predicted. ${ }^{7}$ Schnitzer therefore relaxed the condition connecting $a_{0}$ with $a_{2}$ and allowed all three scattering lengths to be parameters. They were chosen to fit the $\pi^{+}$angular distribution at $432 \mathrm{MeV}$ and the magnitude of the $\pi^{+}-p$ inelastic interaction at $470 \mathrm{MeV}^{8}$ Two sets of scattering lengths provided adequate fits. With these sets he successfully predicted the $\pi^{+}$angular distribution at $365 \mathrm{MeV}$ and predicted the total cross sections for all the single-pion-production reactions up to about $500 \mathrm{MeV}$.

The so-called isobar models provide calculations of the effects of final-state $\pi-N$ interactions on the energy distribution of the outgoing particles and on the branching ratios in single- $\pi$-production reactions but do not yield information about absolute cross sections. Lindenbaum and Sternheimer first introduced the isobar model into the analysis of pion production..$^{9}$ For the $\pi \pi N$ final state either of the pions may form a resonant system with the nucleon. The observed energy spectrum of a pion is the sum of two spectra; that of a pion originating from the decay of the isobar and that of a pion recoiling against the isobar. Bergia, Bonsignori, and Stanghellini observed that the amplitudes for these two possibilities should be summed, not the intensities as Lindenbaum and Sternheimer had done. ${ }^{10}$ At low energies, where the regions of the pion-energy spectrum corresponding to the two processes overlap, the interference terms produce a considerable difference between the predictions by Lindenbaum and Sternheimer and those by Bergia et al. Both calculations assume isotropic production and decay of the isobar. Recently, Olsson and Yodh have extended the model of Bergia et al. by inclusion of the $P$-wave decay of the isobar and the requirements of Bose statistics. ${ }^{11}$ The predictions by Olsson and Yodh compare well with the existent data on pion production in the $\pi^{+}-p$ collisions. Olsson and

${ }^{7}$ Barry C. Barish, Richard J. Kurz, Paul G. McManigal, Victor Perez-Mendez, and Julius Solomon, Phys. Rev. Letters 6, 297 (1961).

${ }^{8}$ Howard J. Schnitzer, Phys. Rev. 125, 1059 (1962).

${ }^{9}$ S. J. Lindenbaum and R. B. Sternheimer, Phys. Rev. 105, 1874 (1957); 106, 1107 (1957); 109, 1723 (1958).

${ }^{10} \mathrm{~S}$. Bergia, F. Bonsignori, and A. Stanghellini, Nuovo Cimento 16, 1073 (1960).

${ }_{11}$ M. Olsson and G. B. Yodh, Phys. Rev. Letters 10, 353 (1963). 
TABLE I. $\pi^{+}$differential cross sections.

\begin{tabular}{|c|c|c|c|c|c|c|c|c|c|c|}
\hline \multirow[b]{2}{*}{$\begin{array}{c}\theta \\
(\mathrm{deg})\end{array}$} & \multirow[b]{2}{*}{$\begin{array}{c}T \\
(\mathrm{MeV})\end{array}$} & \multicolumn{4}{|c|}{ Incident $\pi^{-}$energy $(365 \mathrm{MeV})$} & \multirow[b]{2}{*}{$\begin{array}{c}T \\
(\mathrm{MeV})\end{array}$} & \multicolumn{3}{|c|}{ Incident $\pi^{--}$energy $(432 \mathrm{MeV})$} & \multirow[b]{2}{*}{$\begin{array}{c}d^{2} \sigma / d T^{*} d \Omega^{*} \\
(\mu \mathrm{b} / \mathrm{sr}-\mathrm{MeV})\end{array}$} \\
\hline & & $\begin{array}{c}d^{2} \sigma / d T d \Omega \\
(\mu \mathrm{b} / \mathrm{sr}-\mathrm{MeV})\end{array}$ & $\begin{array}{c}T^{*} \\
(\mathrm{MeV})\end{array}$ & $\begin{array}{c}\theta^{*} \\
(\mathrm{deg})\end{array}$ & $\begin{array}{c}d^{2} \sigma / d T^{*} d \Omega^{*} \\
(\mu \mathrm{b} / \mathrm{sr}-\mathrm{MeV})\end{array}$ & & $\begin{array}{c}d^{2} \sigma / d T d \Omega \\
(\mu \mathrm{b} / \mathrm{sr}-\mathrm{MeV})\end{array}$ & $\begin{array}{c}T^{*} \\
(\mathrm{MeV})\end{array}$ & $\begin{array}{c}\theta^{*} \\
(\mathrm{deg})\end{array}$ & \\
\hline $20 \pm 7$ & $\begin{array}{c}78 \pm 8 \\
100 \pm 11 \\
126 \pm 11 \\
152 \pm 14\end{array}$ & $\begin{array}{l}4.0 \pm 0.3 \\
3.5 \pm 0.3 \\
2.6 \pm 0.3 \\
1.7 \pm 0.1\end{array}$ & $\begin{array}{l}36 \pm 5 \\
50 \pm 6 \\
67 \pm 7 \\
84 \pm 9\end{array}$ & $\begin{array}{l}33 \\
32 \\
31 \\
30\end{array}$ & $\begin{array}{l}2.6 \pm 0.2 \\
2.3 \pm 0.2 \\
1.8 \pm 0.2 \\
1.1 \pm 0.1\end{array}$ & $\begin{array}{c}54 \pm 6 \\
80 \pm 8 \\
112 \pm 12 \\
138 \pm 14 \\
167 \pm 19\end{array}$ & $\begin{array}{l}6.0 \pm 0.6 \\
7.0 \pm 0.5 \\
6.5 \pm 0.4 \\
4.3 \pm 0.3 \\
2.7 \pm 0.2\end{array}$ & $\begin{array}{l}19 \pm 3 \\
34 \pm 5 \\
53 \pm 6 \\
70 \pm 8 \\
88 \pm 9\end{array}$ & $\begin{array}{l}38 \\
34 \\
33 \\
32 \\
31\end{array}$ & $\begin{array}{l}3.4 \pm 0.3 \\
4.2 \pm 0.3 \\
4.1 \pm 0.3 \\
2.8 \pm 0.2 \\
1.8 \pm 0.2\end{array}$ \\
\hline 50 土7 & $\begin{array}{c}53 \pm 7 \\
73 \pm 9 \\
99 \pm 12 \\
119 \pm 13 \\
141 \pm 16\end{array}$ & $\begin{array}{l}3.0 \pm 0.2 \\
2.9 \pm 0.2 \\
2.5 \pm 0.2 \\
1.8 \pm 0.2 \\
1.0 \pm 0.1\end{array}$ & $\begin{array}{c}34 \pm 4 \\
50 \pm 5 \\
69 \pm 7 \\
85 \pm 8 \\
103 \pm 10\end{array}$ & $\begin{array}{l}78 \\
75 \\
73 \\
71 \\
71\end{array}$ & $\begin{array}{l}2.3 \pm 0.2 \\
2.3 \pm 0.2 \\
2.0 \pm 0.2 \\
1.5 \pm 0.2 \\
0.8 \pm 0.1\end{array}$ & $\begin{array}{c}55 \pm 7 \\
79 \pm 9 \\
103 \pm 11 \\
124 \pm 12 \\
161 \pm 16\end{array}$ & $\begin{array}{l}5.0 \pm 0.4 \\
4.2 \pm 0.3 \\
4.4 \pm 0.5 \\
3.4 \pm 0.3 \\
1.3 \pm 0.2\end{array}$ & $\begin{array}{c}35 \pm 4 \\
53 \pm 6 \\
71 \pm 8 \\
87 \pm 8 \\
116 \pm 10\end{array}$ & $\begin{array}{l}81 \\
77 \\
75 \\
74 \\
72\end{array}$ & $\begin{array}{l}3.9 \pm 0.3 \\
3.3 \pm 0.2 \\
3.5 \pm 0.4 \\
2.7 \pm 0.2 \\
1.0 \pm 0.1\end{array}$ \\
\hline $80 \pm 7$ & $\begin{array}{c}33 \pm 2 \\
50 \pm 4 \\
73 \pm 7 \\
94 \pm 9 \\
116 \pm 12\end{array}$ & $\begin{array}{l}2.0 \pm 0.3 \\
2.1 \pm 0.2 \\
1.5 \pm 0.1 \\
0.8 \pm 0.1 \\
0.3 \pm 0.04\end{array}$ & $\begin{array}{r}37 \pm 1 \\
53 \pm 3 \\
76 \pm 5 \\
96 \pm 7 \\
119 \pm 9\end{array}$ & $\begin{array}{l}113 \\
109 \\
106 \\
105 \\
104\end{array}$ & $\begin{array}{l}2.2 \pm 0.3 \\
2.2 \pm 0.2 \\
1.5 \pm 0.1 \\
0.8 \pm 0.1 \\
0.3 \pm 0.04\end{array}$ & $\begin{array}{c}33 \pm 3 \\
56 \pm 5 \\
74 \pm 7 \\
97 \pm 10 \\
114 \pm 11\end{array}$ & $\begin{array}{l}2.4 \pm 0.3 \\
3.6 \pm 0.3 \\
2.9 \pm 0.2 \\
2.2 \pm 0.2 \\
1.2 \pm 0.1\end{array}$ & $\begin{array}{r}39 \pm 2 \\
61 \pm 4 \\
79 \pm 6 \\
102 \pm 7 \\
118 \pm 9\end{array}$ & $\begin{array}{l}116 \\
111 \\
109 \\
107 \\
106\end{array}$ & $\begin{array}{l}2.6 \pm 0.3 \\
3.8 \pm 0.3 \\
3.0 \pm 0.2 \\
2.2 \pm 0.2 \\
1.2 \pm 0.1\end{array}$ \\
\hline $110 \pm 7$ & $\begin{array}{l}30 \pm 2 \\
47 \pm 4 \\
62 \pm 6 \\
77 \pm 9\end{array}$ & $\begin{array}{l}1.8 \pm 0.3 \\
1.5 \pm 0.1 \\
0.9 \pm 0.1 \\
0.4 \pm 0.04\end{array}$ & $\begin{array}{r}52 \pm 3 \\
73 \pm 4 \\
92 \pm 6 \\
111 \pm 9\end{array}$ & $\begin{array}{l}137 \\
134 \\
132 \\
131\end{array}$ & $\begin{array}{l}2.5 \pm 0.4 \\
2.0 \pm 0.2 \\
1.1 \pm 0.1 \\
0.5 \pm 0.05\end{array}$ & $\begin{array}{c}33 \pm 3 \\
50 \pm 5 \\
66 \pm 7 \\
86 \pm 9 \\
107 \pm 11\end{array}$ & $\begin{array}{l}1.9 \pm 0.2 \\
1.8 \pm 0.1 \\
1.8 \pm 0.1 \\
0.8 \pm 0.1 \\
0.2 \pm 0.04\end{array}$ & $\begin{array}{c}60 \pm 3 \\
82 \pm 5 \\
102 \pm 5 \\
127 \pm 9 \\
153 \pm 11\end{array}$ & $\begin{array}{l}138 \\
135 \\
134 \\
133 \\
132\end{array}$ & $\begin{array}{l}2.7 \pm 0.3 \\
2.4 \pm 0.2 \\
2.4 \pm 0.2 \\
1.1 \pm 0.1 \\
0.3 \pm 0.05\end{array}$ \\
\hline
\end{tabular}

Yodh present no comparison of their predictions with $\pi$ energy distributions in $\pi^{-}-p$ interactions, and they indicate that an isobar model without $\pi-\pi$ interaction does not agree with the data on $\pi^{+} \pi^{-} n$.

\section{RESULTS}

Our objective for each of the single-particle measurements was the determination of the differential cross section $d^{2} \sigma(T, \theta) / d T d \Omega$, where $T$ and $\theta$ are the labsystem kinetic energy and angle of the particle of interest and $\Omega$ is the lab-system solid angle. In each experiment the energy distribution of the particle was measured at a set of discrete angles. The design of each detection system and the angles and energies at which measurements were made were chosen to cover the kinematically available range of the variables $T$ and $\theta$.

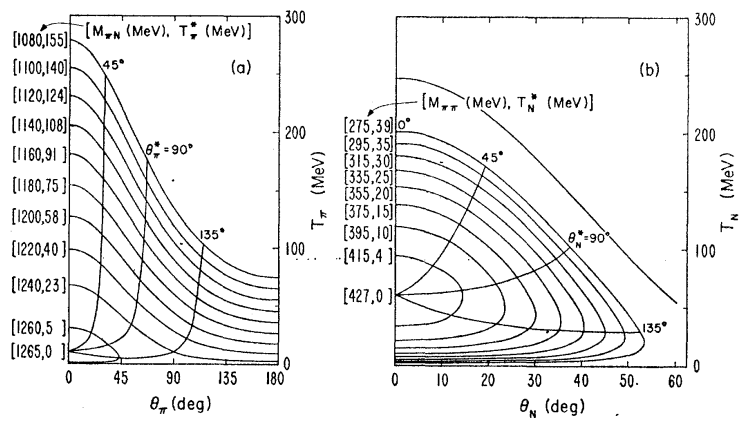

FIG. 1. Laboratory-system kinematics for final-state particles in $\pi N \rightarrow \pi \pi N$. Contours of constant c.m.-system kinetic energy $T^{*}$ and angle $\theta^{*}$ are plotted vs laboratory-system kinetic energy $T$ and angle $\theta$. (a) Final-state $\pi$ for $432-\mathrm{MeV}$ incident $\pi$ kinetic energy. (b) Final-state $N$ for $375-\mathrm{MeV}$ incident $\pi$ kinetic energy. The curve for $\pi N \rightarrow \pi N$ is also shown.
A diagram of the lab-system kinematics for a final state $\pi$ and for the final state $N$ in the reaction $\pi+N \rightarrow$ $\pi+\pi+N$ is presented in Fig. 1. The simplest means of interpreting these diagrams is to consider the single particle as recoiling against the composite system of the other two final-state particles. Contours of constant energy in the over-all c.m. system (i.e., constant energy of the composite system in its two-body rest system, which we denote by $M_{i j}$ ) and constant over-all c.m.system angle are indicated in the diagrams.

In interpreting the results, it is desirable to transform the measured distributions to the over-all c.m. system and present the results as c.m.-system energy distributions at constant c.m.-system angles. This has been done in the $\pi^{+}$case but is inconvenient in the case of the nucleons due to the nonlinear nature of the transformation apparent in Fig. 1(b).

Absolute normalization of the differential cross sections was obtained in the three experiments by monitoring the incident- $\pi^{-}$flux. Corrections were made for the $\mu^{-}$and $e^{-}$components of the beam (5 to 10\%) and for inaccuracies of the monitor systems at high-beam intensities ( 2 to $10 \%$ ) due to the cyclotron duty cycle.

The errors quoted include, in addition to the statistical error, those arising from the subtraction of background, the uncertainties in the normalization, the uncertainties in corrections applied to the data, and uncertainties in the determination of the energy-solidangle acceptance of the detection systems.

\section{A. $\pi^{+}$Differential Distributions}

The $\pi^{+}$in the reaction $\pi^{-}+p \rightarrow \pi^{+}+\pi^{-}+n$ was detected by a system of scintillation-counter telescopes and a magnetic spectrometer at incident- $\pi^{-}$energies of 
TABLE II. Inelastic proton differential cross sections [values of $d^{2} \sigma / d T d \Omega$ in units of $\left.\mu \mathrm{b} /(\mathrm{sr}-\mathrm{MeV})\right]$.

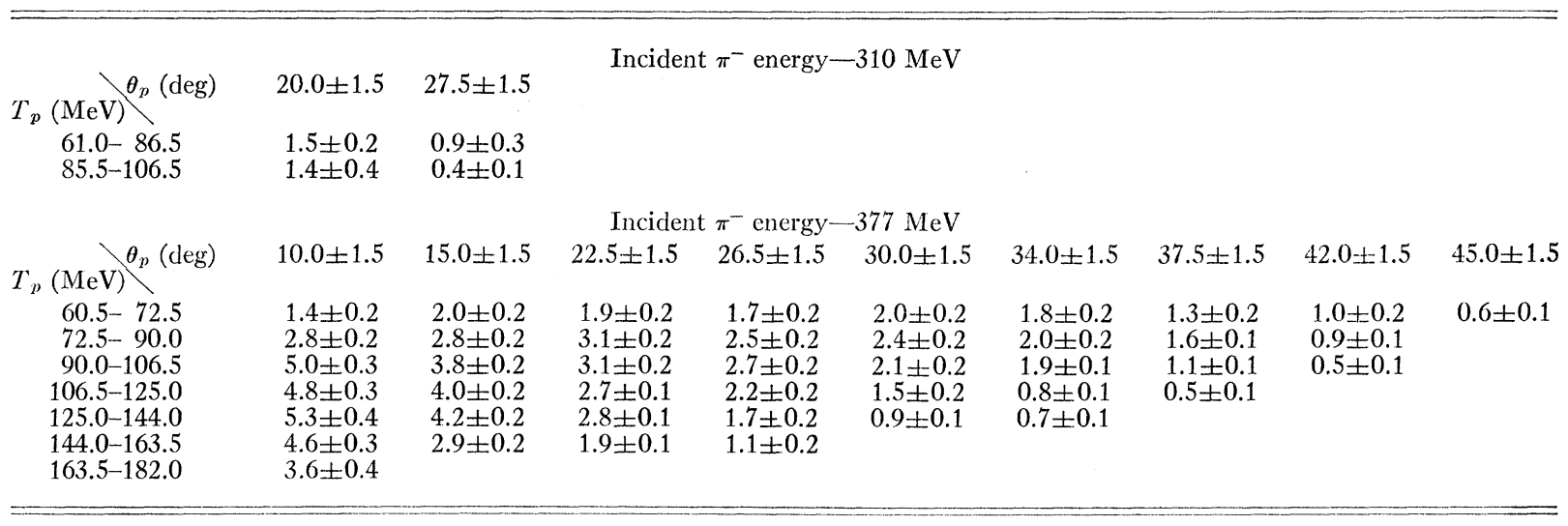

365 and $432 \mathrm{MeV}$. The spectrometer selected positively charged particles. Therefore, protons from other reactions of the $\pi^{-}-p$ incident state were eliminated from the system by a range criterion. The measured values of the $\pi^{+}$differential cross section for 365 and $432-\mathrm{MeV}$ incident $-\pi^{-}$energy are tabulated in Table I. For each $\pi^{+}$lab-system energy, angle, and cross section, the corresponding c.m.-system quantities, $T^{*}$, $\theta^{*}$, and $d^{2} \sigma / d T^{*} d \Omega^{*}$, are listed.

A feature of the $\pi^{+}$differential distributions is the low-energy peaking of the energy spectra. A typical spectrum, the c.m. distribution for the 50-deg labsystem data (average $\theta^{*}=73 \mathrm{deg}$ ) at $365-\mathrm{MeV}$ incident energy, is plotted in Fig. 2(a). Also drawn are a phasespace distribution and a total isotopic spin $\frac{1}{2}$ and $\frac{3}{2}$ energy distributions calculated with the isobar model of Bergia et al. The angular distributions at a constant $T^{*}$ (i.e., constant $M_{\pi N}$ ) shown in Fig. 2(b) are quite isotropic except for values of $T^{*}$, which correspond to $M_{\pi N}$ approaching the $(3,3)$ resonance energy of 1238 $\mathrm{MeV}$ where the $\pi^{+}$becomes forward peaked.

\section{B. Proton Differential Distributions}

In order to obtain data on the $\pi^{-} \pi^{0} p$ reaction, proton distributions were measured with a scintillationcounter telescope at incident- $\pi^{-}$energies of 310 and $377 \mathrm{MeV}$. Inelastic protons were differentiated from elastic protons, elastic pions, and inelastic pions by a combination of range, $d E / d x$, time of flight, and a conjugate elastic-pion counter. No differentiation between protons from $\pi^{-} \pi^{0} p$ and protons from the final-state $\pi^{-}+\gamma+p$ was possible with the experimental apparatus. The measurements by Blokhintseva et al. at $340-\mathrm{MeV}$ incident- $\pi^{-}$energy indicate that the two reactions are comparable in magnitude at that energy. ${ }^{12}$ An estimate based upon the $340-\mathrm{MeV}$ data indicates that the mag-

12 T. D. Blokhintseva, V. G. Grebinnik, V. A. Zhukov, G, Libman, L. L. Nemenov, G. I. Selivanov, and Y. Jung-Fang, Zh. Eksperim. i Teor. Fiz. 44, 116 (1963) [English transl: Soviet Phys.-JETP 17, 80 (1963)]. nitude of the $\pi^{-} p \gamma$ reaction at $377 \mathrm{MeV}$ is $25 \%$ of the total inelastic-proton yield (see Sec. IID2).

The measured values of the inelastic-proton differential cross section for 310 and $377 \mathrm{MeV}$ are tabulated in Table II. At $310-\mathrm{MeV}$ incident- $\pi^{-}$energy only four selected points were measured. The intention was to extract the total cross section but not the details of the differential distributions.

To illustrate the nature of the differential distributions, the proton-energy distribution of the 15-deg data and the angular distribution of the data in the 60.5- to $72.5-\mathrm{MeV}$ proton energy channel at $377-\mathrm{MeV}$ incident energy are plotted in Fig. 3. Also drawn are phasespace distributions of both of these quantities for $\pi^{-} \pi^{0} p$. Whereas, the angular distribution at a fixed energy agrees with the phase-space distribution, the inelastic-proton energy distributions have a high-energy peaking with respect to phase-space distribution.

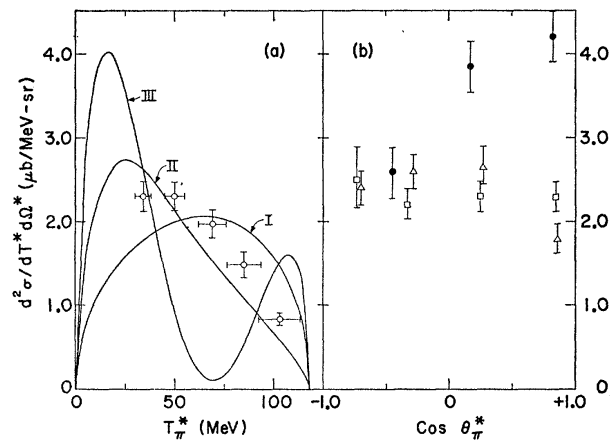

FIG. 2. $\pi^{+}$c.m.-system differential distributions for $\pi^{+} \pi^{-} n$. (a) Distribution in kinetic energy $T^{*}$ at average angle, $\theta^{*}=73 \mathrm{deg}$ at $365-\mathrm{MeV}$ incident $\pi^{-}$energy. The solid curves are: $\mathrm{I}$-invariant phase-space distribution; II-isobar model distribution in the total isotopic spin, $I=\frac{1}{2}$ state; III-isobar model distribution in the $I=\frac{3}{2}$ state (Ref. 10 ). All curves are normalized to the integral of the measured distribution over energy. (b) Distribution in $\cos \theta^{*}$ at several values of $T^{*}$. $\bullet-T^{*}=35 \mathrm{MeV}\left(M_{\pi N}=1225 \mathrm{MeV}\right)$ at $432 \mathrm{MeV} ; \triangle-T^{*}=90 \mathrm{MeV}\left(M_{\pi N}=1160 \mathrm{MeV}\right)$ at $432 \mathrm{MeV}$; and $\square-T^{*}=50 \mathrm{MeV}\left(M_{\pi N}=1160 \mathrm{MeV}\right)$ at $365 \mathrm{MeV}$. 
TABLE III. Inelastic neutron differential cross sections [values of $d^{2} \sigma / d T d \Omega$ in units of $\mu \mathrm{b} /(\mathrm{sr}-\mathrm{MeV})$ ].

\begin{tabular}{|c|c|c|c|c|c|c|c|c|c|c|}
\hline $\begin{array}{c}\theta_{n}(\mathrm{deg}) \\
T \text { interval } \\
(\mathrm{MeV})\end{array}$ & $10 \pm 2$ & $15 \pm 2$ & $20 \pm 2$ & $25 \pm 2$ & $30 \pm 2$ & $35 \pm 2$ & $40 \pm 2$ & $45 \pm 2$ & $50 \pm 2$ & $55 \pm 2$ \\
\hline \multicolumn{11}{|c|}{$\pi^{-}+p \rightarrow \pi^{+}+\pi^{-}+n$ at incident $\pi^{-}$energy $-374 \mathrm{MeV}$} \\
\hline $\begin{array}{c}20-31 \\
31-42 \\
42-53 \\
53-66 \\
66-79 \\
79-90 \\
90-101 \\
101-111 \\
111-125 \\
125-142 \\
142-163 \\
163-190 \\
190-225\end{array}$ & $\begin{array}{r}11.0 \pm 1.1 \\
19.0 \pm 0.9 \\
25.8 \pm 0.8 \\
32.8 \pm 0.8 \\
32.2 \pm 0.8 \\
34.1 \pm 1.0 \\
29.7 \pm 0.8 \\
23.9 \pm 0.8 \\
20.0 \pm 0.7 \\
14.4 \pm 0.5 \\
9.9 \pm 0.5 \\
5.8 \pm 0.5 \\
3.2 \pm 0.3\end{array}$ & & $\begin{array}{r}9.9 \pm 1.6 \\
12.2 \pm 1.3 \\
16.1 \pm 0.8 \\
20.6 \pm 0.8 \\
21.7 \pm 0.9 \\
20.6 \pm 1.0 \\
18.2 \pm 0.9 \\
17.2 \pm 1.0 \\
13.0 \pm 0.8 \\
7.8 \pm 0.6 \\
4.7 \pm 0.4 \\
2.7 \pm 0.5 \\
1.0 \pm 0.3\end{array}$ & & $\begin{array}{l}9.1 \pm 0.6 \\
8.0 \pm 0.5 \\
9.7 \pm 0.4 \\
9.4 \pm 0.4 \\
8.5 \pm 0.4 \\
7.9 \pm 0.5 \\
5.5 \pm 0.4 \\
3.9 \pm 0.3 \\
3.8 \pm 0.3 \\
2.7 \pm 0.3 \\
1.6 \pm 0.2\end{array}$ & $\begin{array}{l}7.7 \pm 0.8 \\
6.4 \pm 0.6 \\
6.0 \pm 0.4 \\
6.7 \pm 0.4 \\
5.4 \pm 0.4 \\
3.7 \pm 0.5 \\
3.2 \pm 0.4 \\
2.6 \pm 0.4 \\
1.7 \pm 0.2\end{array}$ & $\begin{array}{l}7.4 \pm 0.7 \\
6.0 \pm 0.6 \\
5.2 \pm 0.6 \\
3.3 \pm 0.5 \\
5.0 \pm 0.5 \\
3.3 \pm 0.6\end{array}$ & $\begin{array}{l}7.3 \pm 0.6 \\
5.4 \pm 0.5 \\
4.3 \pm 0.4 \\
3.4 \pm 0.3\end{array}$ & $\begin{array}{l}7.3 \pm 0.5 \\
4.3 \pm 0.4\end{array}$ & \\
\hline \multicolumn{11}{|c|}{$\pi^{-}+p \rightarrow \pi^{0}+\pi^{0}+n$ at incident $\pi^{-}$energy $-374 \mathrm{MeV}$} \\
\hline $\begin{array}{c}20-31 \\
31-42 \\
42-53 \\
53-66 \\
66-79 \\
79-90 \\
90-101 \\
101-111 \\
111-125 \\
125-142 \\
142-163 \\
163-190 \\
190-225\end{array}$ & $\begin{array}{r}7.5 \pm 0.8 \\
8.1 \pm 0.7 \\
11.3 \pm 0.3 \\
14.6 \pm 0.6 \\
16.9 \pm 0.6 \\
16.3 \pm 0.7 \\
13.8 \pm 0.6 \\
11.7 \pm 0.7 \\
11.0 \pm 0.6 \\
7.2 \pm 0.4 \\
4.9 \pm 0.4 \\
1.8 \pm 0.4 \\
1.1 \pm 0.3\end{array}$ & & $\begin{array}{l}4.5 \pm 1.1 \\
6.6 \pm 1.1 \\
7.5 \pm 0.6 \\
9.6 \pm 0.6 \\
9.0 \pm 0.6 \\
8.2 \pm 0.7 \\
7.7 \pm 0.8 \\
5.4 \pm 0.5 \\
4.9 \pm 0.5 \\
3.9 \pm 0.4 \\
1.7 \pm 0.4 \\
0.3 \pm 0.6\end{array}$ & & $\begin{array}{l}2.7 \pm 0.5 \\
3.5 \pm 0.4 \\
4.4 \pm 0.3 \\
4.5 \pm 0.3 \\
3.8 \pm 0.3 \\
3.0 \pm 0.4 \\
3.6 \pm 0.4 \\
2.9 \pm 0.3 \\
2.7 \pm 0.3 \\
1.6 \pm 0.2 \\
0.5 \pm 0.2\end{array}$ & $\begin{array}{l}3.4 \pm 0.6 \\
3.9 \pm 0.5 \\
3.4 \pm 0.4 \\
3.0 \pm 0.4 \\
3.9 \pm 0.3 \\
2.9 \pm 0.4 \\
3.7 \pm 0.4 \\
2.5 \pm 0.5 \\
1.5 \pm 0.3\end{array}$ & $\begin{array}{l}1.8 \pm 0.6 \\
2.2 \pm 0.5 \\
1.5 \pm 0.5 \\
2.3 \pm 0.4 \\
3.4 \pm 0.5 \\
5.7 \pm 0.6 \\
3.4 \pm 0.6\end{array}$ & $\begin{array}{l}3.6 \pm 0.5 \\
3.7 \pm 0.4 \\
2.8 \pm 0.4 \\
4.1 \pm 0.4\end{array}$ & $\begin{array}{l}4.2 \pm 0.4 \\
3.4 \pm 0.4\end{array}$ & \\
\hline \multicolumn{11}{|c|}{$\pi^{-}+p \rightarrow \pi^{+}+\pi^{-}+n$ at incident $\pi^{-}$energy $-417 \mathrm{MeV}$} \\
\hline $\begin{array}{c}20-31 \\
31-42 \\
42-53 \\
53-66 \\
66-79 \\
79-90 \\
90-101 \\
101-111 \\
111-125 \\
125-142 \\
142-163 \\
163-190 \\
190-225\end{array}$ & $\begin{array}{r}11.4 \pm 1.1 \\
14.1 \pm 1.0 \\
23.2 \pm 0.9 \\
30.4 \pm 1.0 \\
35.4 \pm 1.0 \\
36.4 \pm 0.9 \\
34.2 \pm 1.1 \\
30.8 \pm 1.0 \\
24.1 \pm 0.8 \\
19.8 \pm 0.8 \\
13.2 \pm 0.6 \\
9.4 \pm 0.5 \\
5.2 \pm 0.4\end{array}$ & $\begin{array}{r}12.0 \pm 1.4 \\
14.5 \pm 1.3 \\
16.5 \pm 1.4 \\
24.3 \pm 1.3 \\
27.0 \pm 1.4 \\
27.9 \pm 1.3 \\
27.1 \pm 1.7 \\
24.5 \pm 1.5 \\
22.3 \pm 1.3 \\
17.2 \pm 1.1 \\
12.5 \pm 0.9 \\
9.6 \pm 0.7 \\
4.8 \pm 0.5\end{array}$ & $\begin{array}{r}11.8 \pm 1.1 \\
11.0 \pm 1.0 \\
16.0 \pm 0.9 \\
20.7 \pm 0.8 \\
21.0 \pm 0.9 \\
21.1 \pm 0.7 \\
19.3 \pm 1.1 \\
19.5 \pm 1.0 \\
14.9 \pm 0.8 \\
12.6 \pm 0.6 \\
8.1 \pm 0.5 \\
5.5 \pm 0.5\end{array}$ & $\begin{array}{r}8.5 \pm 1.4 \\
8.5 \pm 1.2 \\
12.2 \pm 1.1 \\
13.8 \pm 1.1 \\
12.8 \pm 1.1 \\
11.9 \pm 1.0 \\
13.3 \pm 0.9 \\
11.7 \pm 1.0 \\
9.2 \pm 1.0 \\
7.0 \pm 0.8 \\
4.5 \pm 0.6 \\
3.0 \pm 0.5\end{array}$ & $\begin{array}{r}9.4 \pm 1.4 \\
7.6 \pm 1.1 \\
8.1 \pm 1.1 \\
9.9 \pm 1.0 \\
8.3 \pm 1.0 \\
10.9 \pm 1.3 \\
6.8 \pm 1.3 \\
6.9 \pm 1.2 \\
3.7 \pm 0.9 \\
4.2 \pm 0.7 \\
1.6 \pm 0.6\end{array}$ & $\begin{array}{r}11.3 \pm 1.2 \\
5.5 \pm 1.1 \\
8.7 \pm 1.1 \\
7.7 \pm 0.9 \\
6.8 \pm 1.0 \\
5.9 \pm 1.1 \\
6.3 \pm 1.0 \\
4.6 \pm 0.8 \\
3.0 \pm 0.7 \\
1.3 \pm 0.6\end{array}$ & $\begin{array}{l}8.9 \pm 1.4 \\
4.7 \pm 1.2 \\
6.9 \pm 0.8 \\
6.1 \pm 0.7 \\
5.0 \pm 0.9 \\
5.9 \pm 1.0 \\
5.5 \pm 0.7 \\
2.7 \pm 0.8\end{array}$ & $\begin{array}{l}8.0 \pm 1.4 \\
7.9 \pm 1.1 \\
4.4 \pm 1.0 \\
7.2 \pm 0.8 \\
5.5 \pm 1.0 \\
4.5 \pm 1.1\end{array}$ & $\begin{array}{r}13.3 \pm 1.9 \\
8.1 \pm 1.4 \\
5.7 \pm 1.3 \\
4.5 \pm 1.1\end{array}$ & $6.8 \pm 1.9$ \\
\hline \multicolumn{11}{|c|}{$\pi^{-}+p \rightarrow \pi^{0}+\pi^{0}+n$ at incident $\pi^{-}$energy $-417 \mathrm{MeV}$} \\
\hline $\begin{array}{c}20-31 \\
31-42 \\
42-53 \\
53-66 \\
66-79 \\
79-90 \\
90-101 \\
101-111 \\
111-125 \\
125-142 \\
142-163 \\
163-190 \\
190-225\end{array}$ & $\begin{array}{r}5.3 \pm 0.7 \\
7.6 \pm 0.7 \\
9.9 \pm 0.8 \\
13.2 \pm 0.7 \\
15.7 \pm 0.7 \\
16.0 \pm 0.8 \\
15.5 \pm 0.8 \\
12.4 \pm 0.8 \\
12.5 \pm 0.7 \\
6.8 \pm 0.5 \\
4.3 \pm 0.5 \\
2.3 \pm 0.4 \\
1.4 \pm 0.3\end{array}$ & $\begin{array}{r}4.7 \pm 0.9 \\
5.6 \pm 0.9 \\
7.6 \pm 1.1 \\
10.8 \pm 0.9 \\
11.5 \pm 1.0 \\
11.8 \pm 1.4 \\
10.9 \pm 1.1 \\
10.0 \pm 1.1 \\
8.3 \pm 0.9 \\
5.9 \pm 0.8 \\
4.9 \pm 0.6 \\
2.8 \pm 0.5 \\
1.4 \pm 0.4\end{array}$ & $\begin{array}{r}4.7 \pm 0.8 \\
5.5 \pm 0.7 \\
6.5 \pm 0.7 \\
9.2 \pm 0.6 \\
11.5 \pm 0.6 \\
9.7 \pm 0.8 \\
7.6 \pm 0.8 \\
7.7 \pm 0.7 \\
6.5 \pm 0.6 \\
4.2 \pm 0.5 \\
2.3 \pm 0.5 \\
0.4 \pm 0.4\end{array}$ & $\begin{array}{l}5.0 \pm 1.0 \\
2.2 \pm 0.9 \\
5.5 \pm 0.8 \\
6.6 \pm 0.8 \\
7.9 \pm 0.8 \\
7.4 \pm 0.8 \\
5.5 \pm 0.9 \\
5.3 \pm 0.9 \\
4.9 \pm 0.7 \\
2.0 \pm 0.6 \\
1.7 \pm 0.6 \\
1.2 \pm 0.4\end{array}$ & $\begin{array}{l}3.5 \pm 1.0 \\
2.3 \pm 0.9 \\
4.2 \pm 0.8 \\
2.9 \pm 0.8 \\
4.2 \pm 0.9 \\
5.6 \pm 1.1 \\
6.0 \pm 1.0 \\
5.6 \pm 0.8 \\
3.6 \pm 0.8 \\
1.4 \pm 0.7 \\
1.8 \pm 0.6\end{array}$ & $\begin{array}{l}3.7 \pm 0.9 \\
4.0 \pm 0.7 \\
3.8 \pm 0.9 \\
2.4 \pm 0.7 \\
2.5 \pm 0.8 \\
2.5 \pm 0.8 \\
3.9 \pm 1.0 \\
5.0 \pm 0.8 \\
2.5 \pm 0.7 \\
1.2 \pm 0.6\end{array}$ & $\begin{array}{l}1.3 \pm 1.1 \\
3.0 \pm 0.9 \\
3.0 \pm 0.6 \\
3.6 \pm 0.6 \\
2.9 \pm 0.9 \\
2.1 \pm 0.8 \\
3.1 \pm 0.8 \\
1.8 \pm 0.7\end{array}$ & $\begin{array}{l}5.2 \pm 1.0 \\
3.6 \pm 0.9 \\
4.1 \pm 0.8 \\
4.0 \pm 0.8 \\
5.3 \pm 0.9 \\
0.0 \pm 0.7\end{array}$ & $\begin{array}{l}3.7 \pm 1.4 \\
6.5 \pm 1.1 \\
2.9 \pm 1.1 \\
4.0 \pm 1.1\end{array}$ & $5.7 \pm 1.2$ \\
\hline \multicolumn{11}{|c|}{$\pi^{-}+p \rightarrow \pi^{+}+\pi^{-}+n$ at incident $\pi^{-}$energy $-454 \mathrm{MeV}$} \\
\hline $\begin{array}{c}20-31 \\
31-42 \\
42-55 \\
55-70 \\
70-85 \\
85-105 \\
105-120 \\
120-136\end{array}$ & $\begin{array}{l}21.8 \pm 3.8 \\
22.7 \pm 3.5 \\
15.5 \pm 3.1 \\
27.3 \pm 4.0 \\
35.4 \pm 4.2 \\
40.0 \pm 3.5 \\
27.9 \pm 3.8 \\
28.5 \pm 3.5\end{array}$ & $\begin{array}{l}13.0 \pm 3.6 \\
14.0 \pm 2.8 \\
16.9 \pm 3.6 \\
21.3 \pm 3.2 \\
31.0 \pm 3.8 \\
34.7 \pm 3.5 \\
22.2 \pm 2.4 \\
18.0 \pm 2.6\end{array}$ & $\begin{array}{l}13.6 \pm 3.6 \\
13.6 \pm 3.2 \\
10.2 \pm 2.9 \\
24.0 \pm 3.0 \\
13.9 \pm 3.6 \\
23.8 \pm 2.8 \\
16.2 \pm 3.1 \\
13.0 \pm 2.4\end{array}$ & $\begin{array}{r}13.9 \pm 3.5 \\
7.2 \pm 3.8 \\
14.2 \pm 2.4 \\
17.4 \pm 2.7 \\
12.7 \pm 3.2 \\
7.6 \pm 2.6 \\
8.9 \pm 2.9 \\
7.8 \pm 1.9\end{array}$ & & & & & & \\
\hline
\end{tabular}


TABLE III (continued)

\begin{tabular}{|c|c|c|c|c|c|c|c|c|c|c|}
\hline $\begin{array}{c}\theta_{n}(\mathrm{deg}) \\
T \text { interval } \\
(\mathrm{MeV}) \backslash\end{array}$ & $10 \pm 2$ & $15 \pm 2$ & $20 \pm 2$ & $25 \pm 2$ & $30 \pm 2$ & $35 \pm 2$ & $40 \pm 2$ & $45 \pm 2$ & $50 \pm 2$ & $55 \pm 2$ \\
\hline $\begin{array}{l}136-155 \\
155-180 \\
180-212 \\
212-255\end{array}$ & $\begin{array}{r}18.9 \pm 2.7 \\
15.9 \pm 2.1 \\
10.9 \pm 1.5 \\
5.6 \pm 1.4\end{array}$ & $\begin{array}{r}10.0 \pm 2.6 \\
10.5 \pm 1.8 \\
6.6 \pm 1.3 \\
4.1 \pm 1.0\end{array}$ & $\begin{array}{r}11.3 \pm 2.0 \\
10.7 \pm 1.9 \\
5.8 \pm 1.1\end{array}$ & $\begin{array}{l}8.9 \pm 2.1 \\
4.7 \pm 1.6 \\
4.0 \pm 1.1\end{array}$ & & & & & & \\
\hline \multicolumn{11}{|c|}{$\pi^{-}+p \rightarrow \pi^{0}+\pi^{0}+n$ at incident $\pi^{-}$energy $-454 \mathrm{MeV}$} \\
\hline $\begin{array}{c}20-31 \\
31-42 \\
42-55 \\
55-70 \\
70-85 \\
85-105 \\
105-120 \\
120-136 \\
136-155 \\
155-180 \\
180-212 \\
212-255\end{array}$ & $\begin{array}{r}2.6 \pm 2.3 \\
6.3 \pm 2.5 \\
9.2 \pm 2.4 \\
12.5 \pm 2.4 \\
13.4 \pm 2.8 \\
14.7 \pm 1.8 \\
13.5 \pm 2.3 \\
7.5 \pm 1.9 \\
6.5 \pm 2.1 \\
4.0 \pm 1.6 \\
3.3 \pm 1.1 \\
1.5 \pm 0.9\end{array}$ & $\begin{array}{r}5.1 \pm 2.4 \\
7.2 \pm 1.6 \\
6.8 \pm 2.4 \\
7.5 \pm 2.5 \\
16.9 \pm 2.6 \\
15.5 \pm 2.2 \\
9.2 \pm 1.5 \\
3.4 \pm 1.9 \\
3.9 \pm 1.6 \\
2.9 \pm 1.5 \\
-0.8 \pm 1.1 \\
2.0 \pm 0.7\end{array}$ & $\begin{array}{r}5.5 \pm 2.6 \\
7.0 \pm 1.6 \\
8.9 \pm 2.2 \\
12.9 \pm 1.9 \\
6.3 \pm 2.2 \\
7.4 \pm 1.9 \\
5.4 \pm 2.5 \\
5.2 \pm 1.8 \\
4.8 \pm 1.7 \\
3.0 \pm 1.4 \\
1.2 \pm 1.2\end{array}$ & $\begin{array}{l}5.2 \pm 2.4 \\
6.5 \pm 1.6 \\
3.7 \pm 2.0 \\
5.9 \pm 1.9 \\
9.1 \pm 2.1 \\
6.1 \pm 1.9 \\
3.6 \pm 2.0 \\
8.0 \pm 1.8 \\
0.0 \pm 1.9 \\
2.3 \pm 1.4 \\
0.9 \pm 1.0\end{array}$ & & & & & & \\
\hline
\end{tabular}

\section{Neutron Differential Distributions}

In order to obtain data on the neutrons in the $\pi^{+} \pi^{-} n$ and $\pi^{0} \pi^{0} n$ reactions, neutral particles produced in $\pi^{-}-p$ interactions at 374,417 , and $454 \mathrm{MeV}$ were detected by observing the charged products of their interactions in plastic scintillator. The time-of-flight distribution of the neutral particles was measured and separated into photons, neutrons from the reaction $\pi^{-}+p \rightarrow \pi^{0}+n$, and inelastic neutrons. The neutron distributions from $\pi^{+} \pi^{-} n$ and $\pi^{0} \pi^{0} n$ were separated by the detection of the presence or absence of other charged particles in the final state.

Calculated values of the neutron-detection efficiency were used in the conversion of the neutron time-offlight spectra to differential distributions as a function of energy. The errors quoted do not include any uncertainty in the calculated efficiency, which is esti-

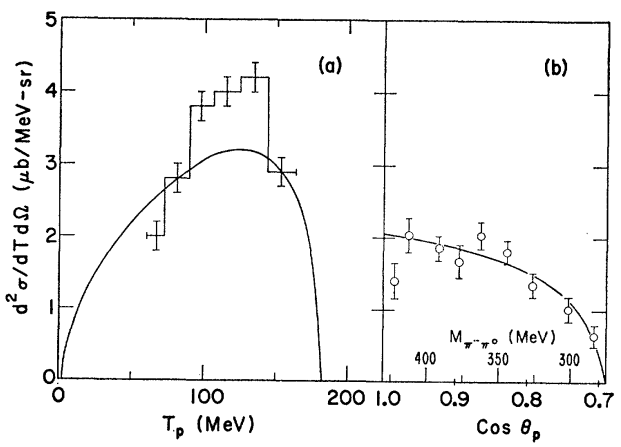

FIG. 3. Inelastic proton lab-system differential distributions at $377-\mathrm{MeV}$ incident- $\pi^{-}$energy. (a) Distribution in kinetic energy $T$ at angle $\theta=15 \mathrm{deg}$. The curve is an invariant phase-space distribution for $\pi^{-} \pi^{0} p$ normalized to the integral of the energy distribution over energy. (b) Distribution in $\cos \theta$ for the energy interval 60.5 to $72.5 \mathrm{MeV}$. A scale of $M_{\pi \pi}$ as a function of $\cos \theta$ at the specified incident- $\pi^{-}$energy and final-state proton energy is also plotted. The curve is the phase-space distribution,

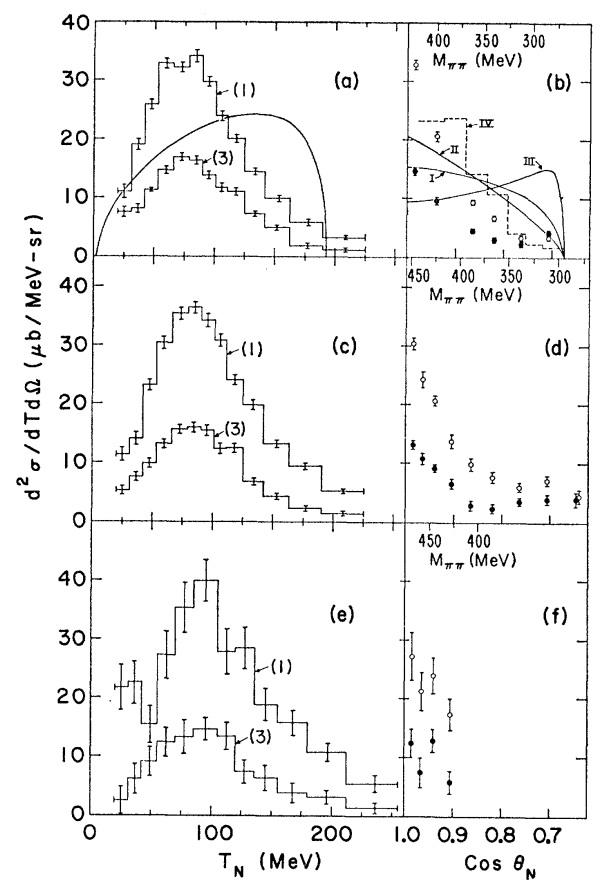

FIG. 4. Neutron lab-system differential distribution. (a), (c), and (e) distribution in kinetic energy $T$ at $\theta=10$ deg for incident $\pi$ energies 374,417 , and $454 \mathrm{MeV}$, respectively. Curve (1) is for $\pi^{+} \pi^{-} n$ and curve (3) for $\pi^{0} \pi^{0} n$. The smooth curve in (a) is the invariant phase-space distribution normalized to the integral of the distribution for $\pi^{+} \pi^{-} n$ over energy. (b), (d), and (f) distribution in $\cos \theta$ for the energy interval 53 to $66 \mathrm{MeV}$. A scale of $M_{\pi \pi}$ as a function of $\cos \theta$ at the specified incident- $\pi^{-}$energy and finalstate neutron energy is also plotted. $0-\pi^{+} \pi^{-} n$; and $-\pi^{0} \pi^{0} n$. The curves in (b) are: I-the phase-space distribution; II-peripheral model distribution for constant $\pi-\pi$ cross section (Ref. 19); III-distribution calculated by using the enhancement factor of $\mathrm{ABC}$ with $a_{0}=2 \mu^{-1}$ and $R=0$ (Ref. 21); and IV-distribution calculated by using the distribution in $M_{\pi \pi}$ of Kirz et al. normalized to the total cross section for $\pi^{+} \pi^{-} n$ at $374 \mathrm{MeV}$ and transformed to the lab system (Ref. 18). With the exception of IV these curves are normalized to the integral of the distribution for $\pi^{+} \pi^{-} n$ over $\cos \theta$ 
TABLE IV. Angular distributions and total cross sections.

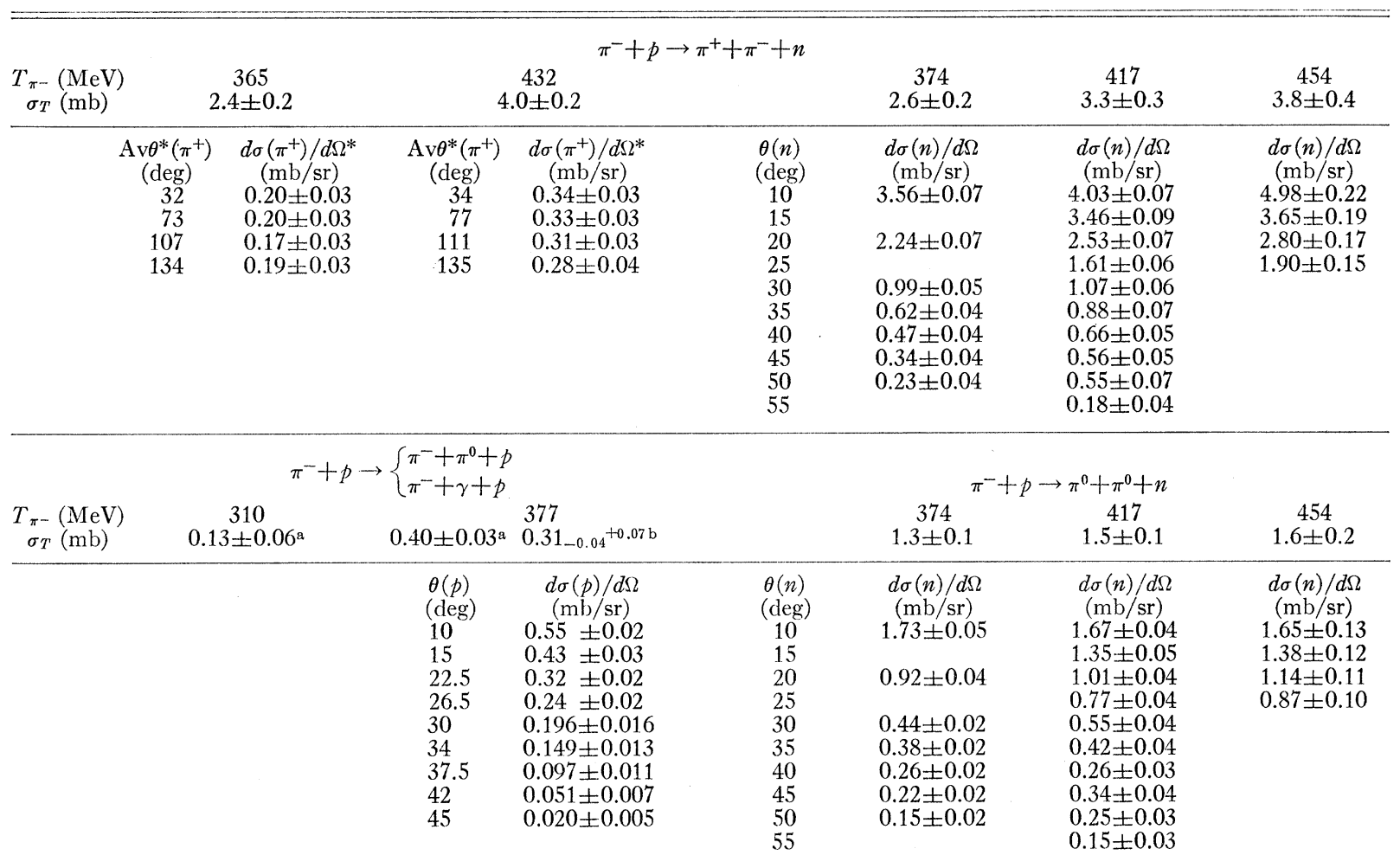

a Total inelastic-proton cross section.

b Estimated $\pi^{-} \pi^{0} p$ cross section (see Sec. IID2).

mated to be less than $10 \%$. A systematic error may exist in the region of the energy distribution near the kinematic limits due to subtraction of the charge-exchange neutrons in the data analysis. The measured values of the neutron differential cross section at 374-, 417-, and 454- $\mathrm{MeV}$ incident- $\pi^{-}$energy are presented in Table III. At $454-\mathrm{MeV}$ incident- $\pi^{-}$energy, data were taken from 10 to 25 deg only.

To illustrate the prominent features of the data, the neutron-energy distribution at 10-deg and the angular distribution of the neutron data in the $53-$ to $66-\mathrm{MeV}$ neutron-energy channel for both reactions and at the three incident energies are plotted in Fig. 4. In Figs. 4(a) and (b) phase-space distributions are also drawn. The other curves are referred to in the discussion of the results below. The data in both reactions and at all incident energies show an enhancement in the distributions in the region of lab-system variables corresponding to the lowest neutron c.m. energy (i.e., highest energy in the $\pi-\pi$ two-body system).

\section{Integrated Cross Sections}

The single-particle differential distributions were integrated between the kinematic limits in energy of the single-pion-production reactions at each angle to yield an angular distribution $d \sigma / d \Omega$. This angular distribu- tion was integrated over angle to obtain a value for the total cross section of the various pion-production reactions. In each case the data do not cover the entire kinematically available range of variables, and the contribution to the integrals of the unmeasured regions was estimated. The uncertainty of these estimates is included in the errors quoted.

\section{Angular Distributions}

In the $\pi^{+}$case the integrations of $d^{2} \sigma / d T^{*} d \Omega^{*}$ over energy were performed in the c.m. system. Although the data for the various values of $\pi^{+}$energy at a fixed lab-system angle do not exactly correspond to the same c.m.-system angle, $\theta^{*}$, the differences from the average $\theta^{*}$ were within the angular resolution of the detection system. The nucleon distributions in each of the pionproduction reactions were integrated over lab-system variables. The values of the angular distributions obtained by integrating $d^{2} \sigma / d T d \Omega$ over energy are tabulated in Table IV and plotted in Fig. 5 .

At both incident- $\pi^{-}$energies, $d \sigma / d \Omega^{*}$ for the $\pi^{+}$is adequately fit by a linear form $d \sigma / d \Omega^{*}=x_{0}+x_{1} \cos \theta^{*}$. The best fits are drawn in Fig. 5(a). Phase-space distributions, normalized to the total cross section, are shown for comparison in the nucleon angulardistribution plots. In both the neutron and the proton 
Fig. 5. Angular distributions after integration over energy. (a) $\pi^{+}$from $\pi^{+} \pi^{-} n$ in the c.m. system. $\triangle$-at $432 \mathrm{MeV}$; O-365 MeV. The solid curves are the best fits to $x_{0}$ $+x_{1} \cos \theta^{*}$ at each energy. The dashed curves are the distributions predicted by Schnitzer for: $\mathrm{I}-\left(a_{0}, a_{1}, a_{2}\right)=\left(0.50,0.07,0.16 \mu^{-1}\right)$; and II- $\left(a_{0}, a_{1}, a_{2}\right)=\left(0.65,0.07,-0.14 \mu^{-1}\right)$ (Ref 8). (b) Proton in the lab system at $377 \mathrm{MeV}$ The curve is the phase-space distribution normalized to the integral of the measured distribution, over $\cos \theta$. (c) Neutron in the lab system. $\bigcirc, \bullet-374 \mathrm{MeV} ; \Delta, \Delta-$ at $417 \mathrm{MeV}$; and $\square,-$ at $454 \mathrm{MeV}$. The open points are for $\pi^{+} \pi^{-} n$ and the solid points for $\pi^{0} \pi^{0} n$. The curves are phase-space distributions normalized to the total cross section at $374 \mathrm{MeV}$; (1) - for $\pi^{+} \pi^{-} n$; and (3) -for $\pi^{0} \pi^{0} n$.
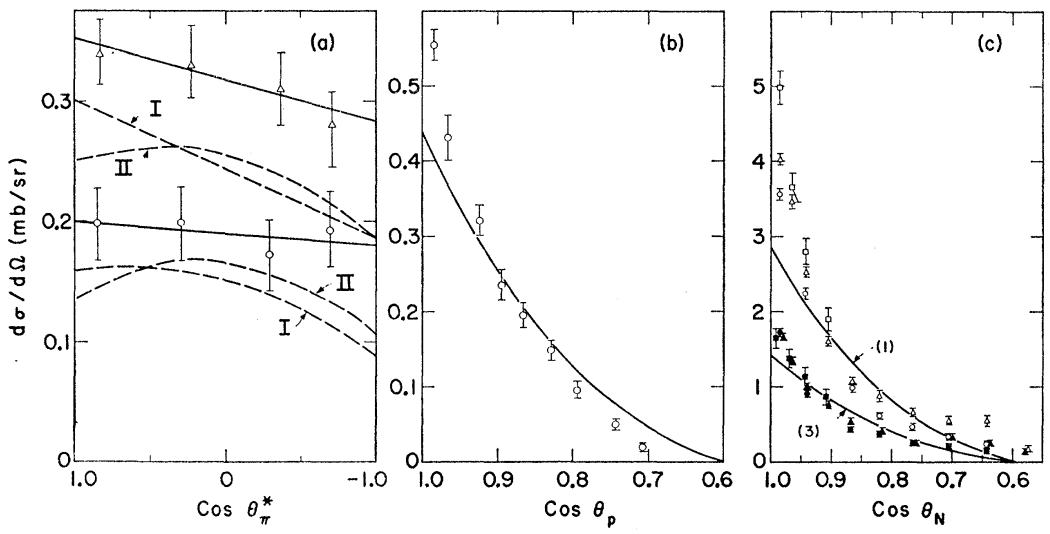

cases the lab-system angular distribution is more forward peaked than a phase-space distribution.

\section{Total Cross Sections}

The total cross sections were obtained by integration over angle of the angular distributions, with three exceptions noted below. In the $\pi^{+}$case the best linear fit was used for the angular distribution.

The contribution of the $\pi^{-} p \gamma$ reaction to the total

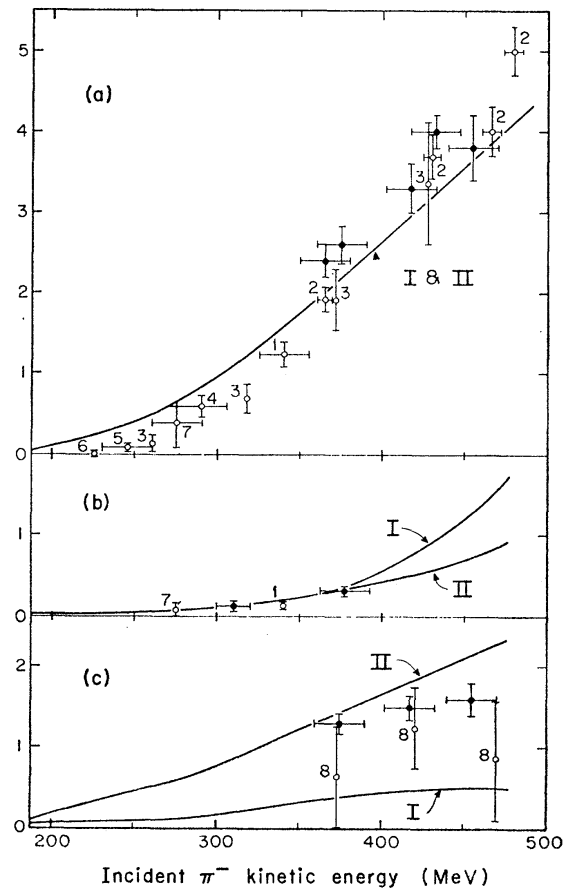

FIg. 6. Total cross sections for single-pion-production reactions below $500 \mathrm{MeV}$. (a) $\pi^{+} \pi^{-} n$; (b) $\pi^{-} \pi^{0} p$; and (c) $\pi^{0} \pi^{0} n$. In each case the curves are the predictions by Schnitzer for solution sets I and II, respectively. The solid points represent data from the present work and the open points represent data from: 1 , Ref. $12 ; 2$, Ref. 18 ; 3, Ref. 3; 4, Ref. 13; 5, Ref. 14; 6, Ref. 15; 7, Ref. 16; 8, Ref. 17. inelastic-proton cross section at $377 \mathrm{MeV}$ was estimated by assuming a phase-space energy dependence of the total cross section for the reaction. The total available phase space at $377 \mathrm{MeV}$ is $24 \%$ greater than at 340 $\mathrm{MeV}$. The quoted cross section at $340 \mathrm{MeV}\left(0.09_{-0.06}+0.03\right.$ $\mathrm{mb}$ ) includes only events in which the $\gamma$ energy exceeds $100 \mathrm{MeV} .{ }^{12}$ This cutoff includes $77 \%$ of the available phase space at $340-\mathrm{MeV}$ incident- $\pi^{-}$energy. Likewise at $377 \mathrm{MeV}$, the cutoff at the kinematic limit of the $\pi^{-} \pi^{0} p$ reaction used here in integrating over the proton energy includes $63 \%$ of the phase space available to the $\pi^{-} p \gamma$ reaction. The net estimated contribution of the $\pi^{--} p \gamma$ reaction to the total inelastic-proton cross section is thus $0.09-0.06{ }^{+0.03} \mathrm{mb}$. This has been subtracted from the total cross section obtained by integration over the inelastic-proton distributions to yield the estimated cross section for the $\pi^{-} \pi^{0} p$ reaction.

For the proton data at $310 \mathrm{MeV}$, only an upper limit on the total cross section for $\pi^{-} \pi^{0} p$ was estimated. The small magnitude of the proton yield and its uncertainty did not make an attempt to estimate the separate contributions of $\pi^{-} \pi^{0} p$ and the $\pi^{-} p \gamma$ reaction worthwhile. A phase-space distribution for $\pi^{-} \pi^{0} p$ was assumed and a total cross section determined from each of the four measured points. The quoted cross section takes into account the extreme values of the individual measurements, including errors.

The total cross sections at $454-\mathrm{MeV}$ incident- $\pi^{-}$energy were estimated from the neutron data by multiplying the total cross sections at $417 \mathrm{MeV}$ by the ratio of (a) the integral over the neutron angular distributions from 10 to $25 \mathrm{deg}$ at $454 \mathrm{MeV}$ to (b) the integral over the same angular region at $417 \mathrm{MeV}$. This method probably underestimates the total cross section.

The measured values of the total cross sections are listed in Table IV. The errors quoted for the total cross sections obtained from the neutron data include the estimated $10 \%$ upper limit on the uncertainty of the calculated neutron-detection efficiency. The total cross sections for the three pion-production reactions are plotted in Fig. 6, along with the available data from 


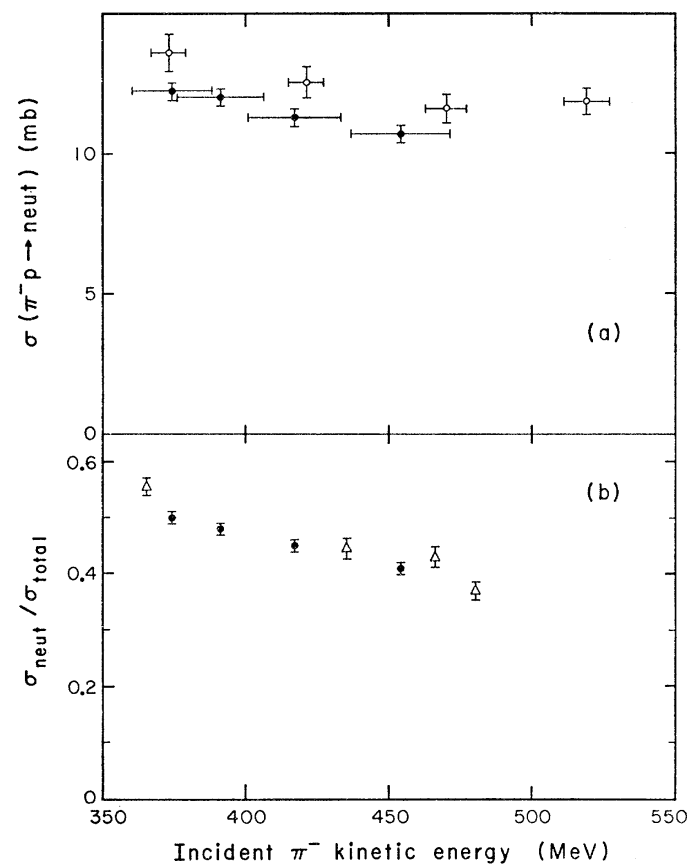

FIg. 7. Total cross section for $\pi^{-}+p \rightarrow$ neutrals. (a) - present experiment; $0-$ Ref. 17. (b) Ratio of total neutral cross section to total $\pi^{-}+p$ cross section. $\bullet-$ present experiment; $\triangle-$ Ref. 18 .

other experiments in this energy region. ${ }^{3,12-18}$ The curves are theoretical predictions and are referred to in the discussion of the results below.

\section{Total Neutral Cross Section}

The determination of the total cross section for the process $\pi^{-}+p \rightarrow$ neutrals was obtained as a byproduct of the electronic data taken during the neutron measurements. In addition to the incident- $\pi^{-}$energies listed above, a short run was made at $391 \mathrm{MeV}$ to measure the total neutral cross section. A correction was made for the detection of the charged products of the interactions of neutral final-state particles in the target surrounding counters $(8 \%)$ ). In addition, the ratio of the total neutral cross section to the total cross section was determined by using the existing data on $\pi^{-}-p$

${ }^{13} \mathrm{Yu}$. A. Batusov, N. P. Bagachev, S. A. Bunyatov, V. M. Sidorov, and Y. A. Yarba, Dokl. Akad. Nauk SSSR 133, 52 (1960) [English transl.: Soviet Phys.-Doklady 5, 731 (1961)].

${ }_{14} \mathrm{Yu}$. A. Batusov, S. A. Bunyatov, V. M. Sidorov, and Y. A. Yarba, Zh. Eksperim. i Teor. Fiz. 39, 1850 (1960) [English transl.: Soviet Phys.-JETP 12, 1290 (1961)].

${ }_{15}$ J. Deahl, M. Derrick, M. Fetkovich, T. Fields, and G. B. Yodh, Proceedings of the 1960 Annual International Conference on High-Energy Physics at Rochester (Interscience Publishers, Inc., New York, 1960), p. 185.

${ }_{16}$ T. D. Blokhintseva, V. G. Grebinnik, V. A. Zhukov, G.

Libman, L. L. Nemenov, G. I. Selivanov, and Y. Jung-Fang, Zh.

Eksperim. i Teor. Fiz. 44, 498 (1963) [English transl.: Soviet

Phys.-JETP 17, 340 (1963)].

${ }_{17}$ René Turley, thèse, Commissariat a L'Energie Atomique Rapport, C.E.A.-2136, 1962 (unpublished).

18 Janos Kirz, Joseph Schwartz, and Robert D. Tripp, Phys. Rev. 130, 2481 (1963).
TABLE V. Total neutral cross sections.

\begin{tabular}{ccc}
\hline \hline $\begin{array}{c}\text { Energy } \\
(\mathrm{MeV})\end{array}$ & $\begin{array}{c}\sigma\left(\pi^{-}+p \rightarrow \text { neutrals }\right) \\
(\mathrm{mb})\end{array}$ & Neutrals/Total \\
\hline $374 \pm 14$ & $12.2 \pm 0.3$ & $0.50 \pm 0.01$ \\
$391 \pm 15$ & $12.0 \pm 0.3$ & $0.48 \pm 0.01$ \\
$417 \pm 16$ & $11.3 \pm 0.3$ & $0.45 \pm 0.01$ \\
$454 \pm 17$ & $10.7 \pm 0.3$ & $0.41 \pm 0.01$ \\
\hline
\end{tabular}

elastic scattering to estimate the number of charged particles scattered by less than $13 \mathrm{deg}$ in the lab system.

The results are presented in Table V. The statistical error in this measurement was negligible $(<0.1 \%)$. The error quoted includes estimated maximum uncertainties in the beam contamination $(1 \%)$, the neutralparticle conversion corrections $(1 \%)$, and the accuracy of the beam-monitor system $(2 \%)$. The data are plotted in Fig. 7 along with other measurements of these quantities in this energy range. ${ }^{17,18}$

\section{E. Discussion}

First we compare the experimental results with the predictions by Schnitzer. ${ }^{8}$ As stated in Sec. I, Schnitzer determined his two sets of $\pi-\pi$ system scattering lengths by fitting the preliminary results of the $\pi^{+}$ angular distributions at $432 \mathrm{MeV}$. The final results reported here have the same qualitative behavior as the preliminary data except that the magnitude of the cross sections is about $15 \%$ higher. The inclusion of systematic errors in the beam-monitor corrections, a more exact evaluation of the energy-solid-angle acceptance of the detection system, and a more detailed treatment of the $\pi^{+}$counting efficiency are responsible for this shift. Thus Schnitzer's $\pi^{+}$angular-distribution curves shown in Fig. 5(a) are too low.

The predictions of the total cross sections for $\pi^{+} \pi^{-} n$, $\pi^{-} \pi^{0} p$, and $\pi^{0} \pi^{0} n$ for both sets of Schnitzer's scattering lengths are shown in Fig. 6. The two sets of solutions give the same result for the $\pi^{+} \pi^{-} n$ reaction that falls slightly below the measurements above $350 \mathrm{MeV}$ and above the measurements below $350 \mathrm{MeV}$. However, the rapid rise of the cross section as a function of energy is present in the predictions. Schnitzer's predictions for the total cross section of the $\pi^{-} \pi^{0} p$ reaction fit the data quite well. The data do not extend to high enough incident energy to differentiate between the two sets of scattering lengths. The results for the $\pi^{0} \pi^{0} n$ reaction do provide a basis for preference for the second set of solutions, which has a negative value of $a_{2}$ and a slightly higher $a_{0}$. Certainly the qualitative behavior of the total cross sections is in agreement with the prediction by the model of Goebel and Schnitzer. Both channels with the $I=0, S$-wave $\pi-\pi$ state available rise rapidly from threshold, whereas the $\pi^{-} \pi^{0} p$ reaction remains low. As the $I=1, P$-wave $\pi-\pi$ interaction becomes significant, the $\pi^{-} \pi^{0} p$ reaction begins to rise and the $\pi^{+} \pi^{-} n$ reaction continues to increase. The $\pi^{0} \pi^{0} n$ reaction, without 
access to the $I=1$ state, levels off as the $S$-wave $\pi-\pi$ interaction falls off.

By use of the peripheral model it is possible to interpret qualitatively the nucleon differential distributions in terms of the $\pi-\pi$ interaction as a function of $\pi-\pi$ system total energy, $M_{\pi \pi \cdot}{ }^{19}$ In this model, $d^{2} \sigma / d T d \Omega$ for the nucleon as a function of lab-system angle at constant energy (which corresponds to constant fourmomentum transfer to the nucleon) is related to the $\pi-\pi$ interaction. So far as the neutron measurements are concerned it is also advantageous to fix the energy, since possible errors in the detection efficiency as a function of neutron energy do not affect this distribution. The measured distribution for nucleon energies of about $60 \mathrm{MeV}$ (invariant momentum transfer equal to 6 in units of the square of the pion mass) are plotted in Figs. 3 and 4 . We observe that there is a strong peaking towards the highest values of $M_{\pi \pi}$ available for the neutrons in the $\pi^{+} \pi^{-} n$ and $\pi^{0} \pi^{0} n$ reactions but not for the proton in the $\pi^{-} \pi^{0} p$ reaction. The peaking of the distributions at low c.m. energy for the neutron (i.e., high $M_{\pi \pi}$ ) has also been observed by Kirz et al. ${ }^{18}$ and Blokhintseva et al. ${ }^{12}$ in the $\pi^{+} \pi^{-} n$ reaction in this energy region. For comparison, the lab-system neutron distribution corresponding to the $M_{\pi \pi}$ distribution of Kirz et al. has been plotted in Fig. 4(b). We also plotted the distribution calculated from the peripheral-model formula, assuming its validity at physical-momentum transfers and using a $\pi-\pi$ cross section that is constant as a function of energy. The presence of the effect in both the $\pi^{+} \pi^{-} n$ and $\pi^{0} \pi^{0} n$ reactions and its absence in the $\pi^{-} \pi^{0} p$ reaction indicate that it is a $I=0$ state effect if the $\pi-\pi$ interaction is responsible for the enhancement. Again in agreement with the data of Kirz et al., as the incident energy is increased the enhancement remains at the highest values of $M_{\pi \pi}$ available, instead of remaining at a constant value of $M_{\pi \pi}$ as would be expected on the basis of a simple resonance behavior in the $\pi-\pi$ system. Evidence form the $\pi^{+}$distributions reported here and from the studies by Kirz et al. indicate that there are strong effects of the nucleon isobar in the $\pi^{+} \pi^{-} n$ final state to compete with the simple peripheral $\pi-\pi$ interaction model. Suggestions have been advanced of a more complex mechanism associated with the interference of $(3,3)$ isobar formation and a peripheral process involving a strong $I=0, S$-wave $\pi-\pi$ interaction, but no quantitative results have been published. ${ }^{11,20}$

The presence of a $I=0, \pi-\pi$ interaction has been proposed as an explanation for the anomaly observed by Abashian et al. (ABC) in $p-d$ collisions. ${ }^{21}$ If an effect of the magnitude required to fit the results of $\mathrm{ABC}$ were

${ }^{19}$ G. F. Chew and F. E. Low, Phys. Rev. 113, 1640 (1959); Charles J. Goebel, Phys. Rev. Letters 1, 337 (1958).

${ }^{20}$ Gyo Takeda, Tohoku University, Sendai, Japan (private communication).

${ }^{21}$ Alexander Abashian, Norman E. Booth, Kenneth M. Crowe, Roger E. Hill, and Ernest H. Rogers, Phys. Rev. 132, 2296, 2305, 2309, 2314 (1963).

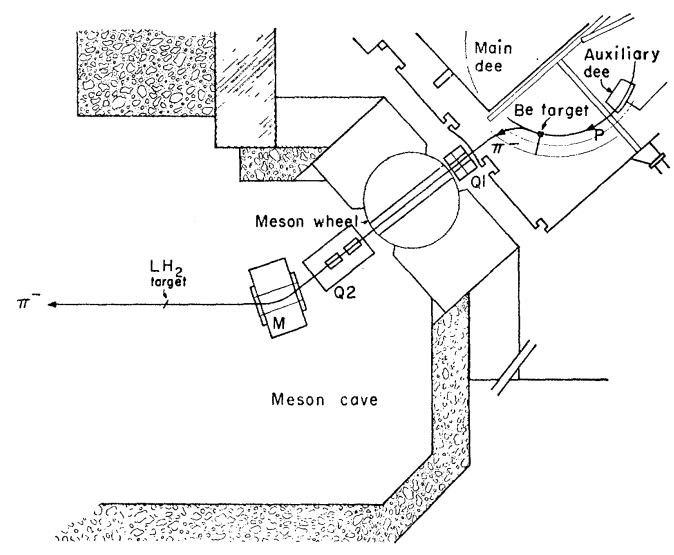

FIG. 8. Plan view of the 184-in.-cyclotron meson-cave experimental area, showing a typical $\pi^{-}$-beam layout.

present in single-pion-production reactions, there would be an enhancement of the neutron distributions in a region corresponding to the lowest values of $M_{\pi \pi}$ available [see Fig. 4(b)]. This behavior is not observed. Unfortunately this exactly corresponds to the regions of greatest uncertainty in the measured neutron distributions, i.e., closest to the charge-exchange neutrons [see Fig. 1(b)]. Because of this uncertainty, together with the fact that the distributions are dominated by a strong enhancement at the opposite extreme of the available $M_{\pi \pi}$ range, it is impossible to make quantitative statements about the ABC effect in $\pi \pi N$ final states. Finally, the absence of a strong manifestation of the $\mathrm{ABC}$ effect observed here is consistent with the results of Kirz et al.

As stated in Sec. I, the calculations by Goebel and Schnitzer make no predictions of the $\pi$ energy distributions. The observed $\pi^{+}$distributions are peaked toward low c.m. energies. Such behavior is most likely explained by an isobar model, since low values of $\pi^{+}$c.m. energy correspond to values of $M_{\pi n}$ in the vicinity of the $(3,3)$ resonance $(1238 \mathrm{MeV})$. In an attempt to fit the $\pi^{+}$data with the aid of the model of Bergia et al., we calculated $d^{2} \sigma / d T^{*} d \Omega^{*}$ for both total isotopic spin $I=\frac{1}{2}$ and $I=\frac{3}{2}$ incident states. These spectra are plotted in Fig. 2(a). The $\pi^{+}$data were then fitted with linear combinations of either of these two distributions and a phase-space distribution. When we attempted to fit the data to just one of the three distributions, the $I=\frac{1}{2}$ incident-state isobarmodel spectrum was by far the best fit, even though the fit was not good. In the attempts to fit linear combinations of the spectra, reasonably good fits were achieved only at $365 \mathrm{MeV}$. Two combinations were modestly successful: the $I=\frac{1}{2}$ or $I=\frac{3}{2}$ incident-state isobar-model distribution with an interfering phase-space background. The main difference was that in the $I=\frac{1}{2}$ case the ratio of the isobar amplitude to the phase-space amplitude was $5 / 1$, whereas for the $I=\frac{3}{2}$ case the corresponding ratio was $\frac{2}{3}$. The $432-\mathrm{MeV}$ data were not fitted well by any of the linear combinations. The nonisotropic angular 
TABLE VI. Beam properties.

\begin{tabular}{cccccccc}
\hline \hline Experiment & Beam size & $\begin{array}{c}\text { Momentum } \\
(\mathrm{MeV} / \mathrm{c})\end{array}$ & $\begin{array}{c}\text { Energy } \\
(\mathrm{MeV})\end{array}$ & $\begin{array}{c}\Delta T \\
(\mathrm{MeV})\end{array}$ & $\begin{array}{c}\mu^{-} \times 100 / \\
\left(\pi^{-}+\mu^{-}+e^{-}\right)\end{array}$ & $\begin{array}{c}e^{-} \times 100 / \\
\left(\pi^{-}+\mu^{-}+e^{-}\right)\end{array}$ & $\begin{array}{c}\text { Average flux } \\
\left(\pi^{-} / \mathrm{cm}^{2}-\mathrm{sec}\right)\end{array}$ \\
\hline$\pi^{+}$ & $4.5 \times 2$ & 485 & 365 & 15 & $4.1 \pm 0.4$ & $\mathrm{~b}$ & $6 \times 10^{3}$ \\
& $4 \times 3$ & 554 & 432 & 15 & $3.8 \pm 0.4$ & $\mathrm{~b}$ & $2 \times 10^{3}$ \\
& 4 & 428 & 310 & 10 & $7.0 \pm 1.0$ & $0.3 \pm 0.3$ & $12 \times 10^{3}$ \\
$n$ & $4 \times 2.5$ & 497 & 377 & 15 & $10.7 \pm 1.0$ & $\mathrm{~b}$ & $4.5 \times 10^{3}$ \\
& & 494 & 374 & 15 & $5.0 \pm 1.0$ & $\mathrm{~b}$ & $4.5 \times 10^{3 \mathrm{c}}$ \\
& & 539 & 417 & 16 & $\mathrm{~d}$ & $\mathrm{~b}$ & $2 \times 10^{3 \mathrm{c}}$ \\
& & 577 & 454 & 17 & $\mathrm{~d}$ & $\mathrm{~b}$ & $0.3 \times 10^{3 \mathrm{c}}$ \\
\hline
\end{tabular}

a Full width at half-maximum at hydrogen target (in $\mathrm{cm}$ ).

b Assumed to be equal within the quoted error to the $310-\mathrm{MeV}$ measurements.

d Auxiliary dee-high-duty-cycle cylotron operation.

distribution of the isobar production noted here [Fig. 2(b) ] and by Kirz et al. may be responsible. The model used here does not consider the angular distribution of the isobar decay, the angular distribution of the isobar production, and the effects of a strong competing $\pi-\pi$ interaction. The model of Olsson and Yodh would eliminate the first shortcoming but, as they point out, the experimental data of Kirz et al., with which our results are in agreement, still is not explained. We can only concur that the situation must be more complex than either an isobar model or the treatment by Goebel and Schnitzer assumes. The analyses according to the work by Bergia et al. and the work of Goebel and Schnitzer are both consistent with the dominance of the $I=\frac{1}{2}$ incident state in single-pion-production reactions in the energy region below $500 \mathrm{MeV}$.

\section{EXPERIMENTAL METHOD AND DATA ANALYSIS}

All three experiments were done with similar $\pi^{-}$-beam setups in the meson cave of the 184-in. cyclotron. A typical beam layout is shown in Fig. 8. Minor changes in the positions of the second quadrupole magnet and the momentum-analyzing magnet were made from run to run. For the neutron measurement, additional shield-

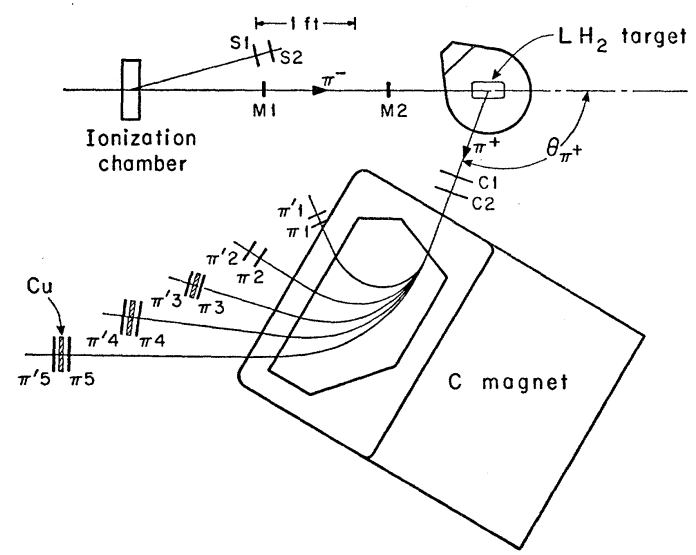

Fig. 9. Diagram of the $\pi^{+}$-detection system. The liquid-hydrogen target was a horizontal cylinder 2 in. in diameter and 4 in. long. ing was used inside the meson cave to reduce the background of neutral particles from the accelerator. The highest $\pi^{-}$-beam energies used in these measurements correspond to the upper limit of that available with the 732-MeV internal-proton-beam energy of the cyclotron. The $\pi^{-}$'s were produced by intercepting the internal proton beam with a $\mathrm{Be}$ target that was 2 in. thick in the beam direction. The pions were deflected outward by the magnetic field of the cyclotron. The trajectories of the $\pi^{-}$up to Q1 were calculated by the CYCLOTRON ORBIT computer program of Good et al. ${ }^{22}$ The current settings of the magnets were determined by the beam optics computer program, OPTIK, ${ }^{23}$ and by suspendedwire measurements. The angle and direction of the deflection in $\mathrm{M}$ were chosen to produce a recombination at the final image of the momentum dispersion introduced by the cyclotron field. The average energy and energy spread of the beam were experimentally checked by integral range measurements in $\mathrm{Cu}$. The beam included $\mu^{-}$and $e^{-}$as well as $\pi^{-}$. The $\mu^{-}$contamination due to $\pi^{-}$decays before $\mathrm{M}$ was determined from the range curves. The contamination due to decays after $\mathrm{M}$ was calculated and combined with the range-curve information to give the total $\mu^{-}$contamination. The electron contamination, measured in one beam setup with a gas Čerenkov counter, was small and was assumed to be the same for all of the $\pi^{-}$beams used. The properties of the beams used in these experiments are listed in Table VI.

\section{A. Experiment I-Measurements of the $\pi^{+}$ in the $\pi^{+} \pi^{-} n$ Mode}

\section{1. $\pi^{+}$Detection System}

A diagram of the $\pi^{+}$-detection system is shown in Fig. 9. The $\pi^{-}$beam was monitored with: (a) two scintillation counters, M1 and M2, located in the beam; (b) an argon-filled ionization chamber; and (c) two scintillation counters, S1 and S2, which detected par-

${ }^{22}$ Joe Good, Morris Pripstein, and Howard S. Goldberg, Lawrence Radiation Laboratory Report UCRL-11044, October 1963 (unpublished).

${ }^{23} \mathrm{~T}$. J. Devlin, Lawrence Radiation Laboratory Report UCRL9727, September 1961 (unpublished). 
ticles scattered from the beam. The three systems were calibrated at low-beam intensities so that the scatter monitor data could be used to determine the error in the beam-counter monitor at high-beam intensities due to the passage of more than one pion through the monitor system within the resolution time of the monitorcoincidence circuit. The average corrections to the counter monitor were $10 \pm 2 \%$ at $365 \mathrm{MeV}$ and $2.5 \pm 0.5 \%$ at $432 \mathrm{MeV}$.

The spectrometer served two purposes. The first was to exclude all negatively charged particles and the second to determine the momentum of positively charged particles. The $\pi^{+}$spectrometer was a nominal 13- by 24-in. C magnet with a 4-in. gap width and specially shaped pole pieces. Data at four lab angles were taken with two magnet-current settings in order to accommodate the range in the $\pi^{+}$momenta, which varied as a function of laboratory-system angle.

The $\pi^{+}$telescope consisted of two scintillation counters, $\mathrm{C} 1$ and $\mathrm{C} 2$, and five sets of two scintillators, $\pi$ and $\pi^{\prime}$. The width of the $\pi$ counters was chosen so that the momentum acceptance of each counter was $\pm 10 \%$ of its central momentum, and the height was chosen in order to detect any particle subject to possible vertical defocusing in the spectrometer. The counter telescope was used for detection of the positively charged particles having range greater than the proton range of the same momentum. Protons were not counted in the $\pi^{\prime}$ counters because of the copper absorber between $\pi$ and $\pi^{\prime}$ sufficiently thick to stop them. A $\pi^{+}$was identified by a M1 M2 C1 C2 $\pi \pi^{\prime}$ coincidence in a conventional fast electronic system.

The configuration and the properties of the combination of the counter telescope and the magnetic spectrometer were determined by suspended-wire measurements. Prior to the run, measurements were made in order to determine the optimum location of the $\pi \pi^{\prime}$ counters for a particular momentum. Since the targetimage location and magnification varied with momentum, it was desirable to place the counter at the image so that the momentum acceptance of the counter was as clean as possible. The counters were positioned along the measured locus of the centers of the various momentum foci. The suspended-wire method was also used to determine the correct maximum vertical size of counters $\mathrm{C} 1$ and $\mathrm{C} 2$ so that no $\pi^{+}$produced by an incident $\pi^{-}$passing through monitor counter M2 could hit the magnet pole pieces.

Subsequent to the run, the solid-angle and energy acceptance for each $\pi \pi^{\prime}$ counter at each lab angle was measured by the suspended-wire method. The acceptance, the product $\Delta T \Delta \Omega$, of the counters was first determined for several points of origin in the target and then was averaged over the whole target. In over $90 \%$ of the measurements the uncertainty in $\Delta T \Delta \Omega$ averaged $4 \%$, but in the other $10 \%$ systematic errors yielded an uncertainty as high as $10 \%$.

\section{Corrections to $\pi^{+}$Data}

A positive particle indirectly produced in $\pi^{-}-p$ collisions is the $e^{+}$arising from the external conversion of photons produced in the decay of $\pi^{0}$ and from internal conversion in the Dalitz decay of $a \pi^{0}$. The main source of the $\pi^{0}$ is from the charge-exchange reaction $\pi^{-}+p \rightarrow$ $\pi^{0}+n$, whose total cross section is about five times the inelastic- $\pi^{0}$-production cross section in the 350 - to $450-\mathrm{MeV}$ energy region. The probability per unit pion energy per steradian of producing an $e^{+}$having the momentum and direction of the $\pi$ due to both conversion and Dalitz positrons was calculated under the assumption that the source of the $\pi^{0}$ was solely the charge-exchange reaction. For the external-conversion case, the differential distribution of photons arising from the charge-exchange $\pi^{0}$ decay was calculated from the measured values of the $\pi^{0}$ distribution. ${ }^{24}$ This was then weighted by the probability of converting the $\gamma$ as a function of $\gamma$ momentum and integrated over all the momenta that may contribute to produce a positronmomentum distribution, $d^{2} \sigma / d p_{e} d \Omega$, which was then transformed to the desired $d^{2} \sigma / d T_{\pi} d \Omega$. A similar calculation was performed for $d^{2} \sigma / d T_{\pi} d \Omega$ due to the Dalitz $e^{+}$.

The calculation agreed well with a measurement of the $e^{+}$contribution due to $\gamma$ conversion at $20 \mathrm{deg}$, which was made by adding more converting material and extrapolating back to the original target thickness. The calculated total $e^{+}$contamination varied from about $15 \%$ at $20 \mathrm{deg}$ to less than $1 \%$ at $110 \mathrm{deg}$ at $365 \mathrm{MeV}$, and from $12 \%$ at $20 \mathrm{deg}$ to less than $1 \%$ at $110 \mathrm{deg}$ at $432 \mathrm{MeV}$. The calculated $e^{+}$contamination was subtracted from the measured spectra. The uncertainty assigned to this correction was that due to the uncertainty in the measurements of the charge-exchange differential cross section.

The path traveled by the particles in the spectrometer was such that 12 to $18 \%$ of the $\pi^{+'}$ s (depending upon

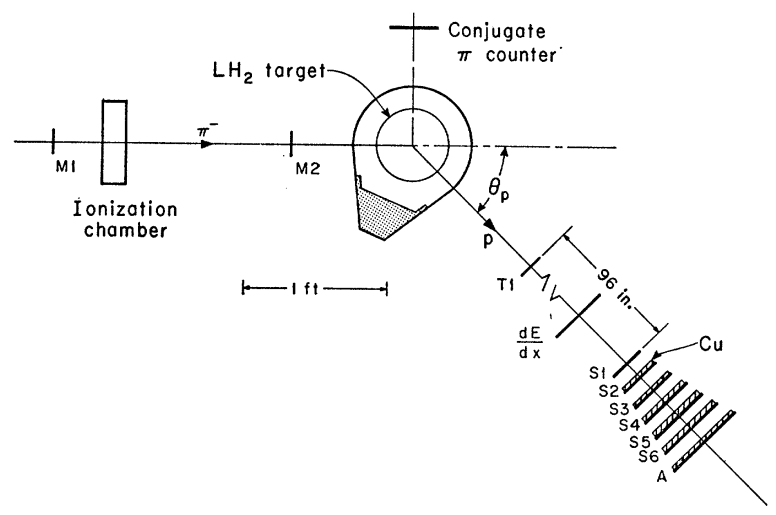

FIG. 10. Diagram of the inelastic-proton-detection system. The liquid-hydrogen target was a vertical cylinder 6 in. in diameter.

${ }^{24}$ John C. Caris, Robert W. Kenney, Victor Perez-Mendez, and Walton A. Perkins, III, Phys. Rev. 121, 893 (1961). 
TABLE VII. Average kinetic energy interval for protons and pions as a function of the stopping counter and the corresponding values of time of flight and $d E / d x$ for these energy intervals.

\begin{tabular}{|c|c|c|c|c|c|c|}
\hline \multirow{2}{*}{$\begin{array}{l}\text { Stopping } \\
\text { counter }\end{array}$} & \multicolumn{2}{|c|}{$\begin{array}{l}\text { Average kinetic energy interval } \\
(\mathrm{MeV})\end{array}$} & \multicolumn{2}{|c|}{$\begin{array}{l}\text { Time-of-flight interval } \\
\text { (nsec) }\end{array}$} & \multicolumn{2}{|c|}{$\begin{array}{l}d E / d x \text { interval } \\
\left(\mathrm{MeV}-\mathrm{cm}^{2} / \mathrm{g}\right)\end{array}$} \\
\hline & Protons & Pions & Protons & Pions & Protons & Pions \\
\hline $\mathrm{S}_{1}$ & $60.5-72.5$ & $19.5-26.0$ & $24.3-27.6$ & $15.2-17.0$ & $9.8-12.3$ & $4.5-5.4$ \\
\hline $\mathrm{S}_{2}$ & $72.5-90.0$ & $26.0-35.0$ & $20.9-24.3$ & $13.2-15.2$ & $7.9-9.8$ & $3.7-4.5$ \\
\hline $\mathrm{S}_{3}$ & $90.0-106.5$ & $35.0-45.0$ & $19.0-20.9$ & $12.5-13.2$ & $6.6-7.9$ & $3.2-3.7$ \\
\hline $\mathrm{S}_{4}$ & $106.5-125.0$ & $45.0-54.5$ & $17.7-19.0$ & $11.8-12.5$ & $5.8-6.6$ & $2.8-3.2$ \\
\hline $\mathrm{S}_{5}$ & $125.0-144.0$ & $54.5-64.0$ & $16.5-17.7$ & $11.1-11.8$ & $5.2-5.8$ & $2.6-2.8$ \\
\hline $\mathrm{S}_{6}$ & $144.0-163.5$ & $64.0-73.0$ & $15.7-16.5$ & $10.7-11.1$ & $4.9-5.2$ & $2.4-2.6$ \\
\hline
\end{tabular}

a Time of flight between counters $\mathrm{T}_{1}$ and $\mathrm{S}_{1}$ (see Fig. 10).

their energy) decayed in flight. It was possible for the resulting $\mu^{+}$'s (a) to end up in the same counter originally intended for the $\pi^{+}$; (b) to pass through some other $\pi$ counter, thus adding extraneous counts to that channel; or (c) to go undetected by any of the $\pi$ counters. The net $\pi^{+}$loss as a function of $\pi^{+}$energy at a given lab-system angle was calculated by a computer program that (a) traced all $\mu$ 's arising from the decay of any $\pi^{+}$through the magnetic field and (b) determined the fraction of these $\mu$ 's that pass through any particular counter. These losses were then weighted by the probability of producing the $\pi^{+}$and summed over all $\pi^{+}$ energies and angles of production. The result turns out to be relatively independent of $\pi^{+}$energy at a given angle. The percentage losses in detection efficiency ranged from $4 \%$ at $20 \mathrm{deg}$ to $9 \%$ at $110 \mathrm{deg}$.

Losses due to nuclear absorption of the $\pi^{+}$in the telescope averaged $6 \%$ for energies above $80 \mathrm{MeV}$ and 2 to $4 \%$ for energies below $80 \mathrm{MeV}$. These were calculated by using Stork's data. ${ }^{25}$ The uncertainty assigned to the correction was taken as $10 \%$ of the correction.

Losses due to multiple Coulomb scattering were calculated by using the Sternheimer ${ }^{26}$ formalism adapted to a rectangular geometry in only the vertical direction, for it was assumed that each particle scattered out of the telescope in the horizontal plane was compensated for by a second particle that was not headed for the telescope, but was scattered into it. The correction was found to be negligible.

\section{B. Experiment II-Measurements of the Proton in the $\pi^{-} \pi^{0} p$ Mode}

\section{Inelastic-Proton-Detection System}

The experimental setup is illustrated in Fig. 10. The incident $\pi^{-}$flux was monitored in an argon-filled ionization chamber. The data at a lab-system angle of $10 \mathrm{deg}$ were taken at lower beam intensities, and scintillation counters M1 and M2 were used to monitor the beam. Data were taken at several larger angles with both

25 Donald Harvey Stork, Phys. Rev. 93, 868 (1954).

${ }^{26}$ R. M. Sternheimer, Rev. Sci. Instr. 25, 1070 (1954). types of monitor to compare the two monitor systems. A range telescope, consisting of six stopping counters with varying thicknesses of copper inserted between scintillation counters, was used to sort particles into intervals of range (energy). The telescope had an angular spread of $1.5 \mathrm{deg}$ and divided pions and protons into the energy intervals given in Table VII.

At a given angle, both elastic protons and pions have a longer range than inelastic protons; however, due to nuclear collisions, a small percentage of each was counted in the stopping counters. Since range alone did not identify protons uniquely, other separating methods had to be employed in conjunction.

As can be seen in Table VII, protons and pions that were stopped in the same counter had different times of flight. The inelastic pions were always at least $4.6 \mathrm{nsec}$ away from the inelastic protons and were clearly eliminated by using a coincidence circuit with a resolving time $2 \mathrm{nsec}$ wider than the inelastic-proton interval. Elastic pions were also clearly separated by the time-offlight method, since they had a time-of-flight separation from inelastic protons that was always greater than 5 nsec for all the angles measured.

Rejection efficiency of the telescope against elastic protons was increased by using an elastic conjugatepion counter. For each angle of scattering for the proton telescope, pions from $\pi^{-}+p \rightarrow \pi^{-}+p$ come off at a definite conjugate angle, whereas the pions from $\pi^{-}+p \rightarrow \pi^{-}+\pi^{0}+p$ have a distribution of angles kinematically available. A counter was placed at the elastic conjugate angle, and no particle was counted in the proton telescope if there was an associated particle in the conjugate counter. This counter had less than a $2 \%$ probability for eliminating inelastic events while eliminating $95 \%$ of elastic events.

Only inelastic protons should be able to satisfy all the above requirements; however, all the other particles also have a smaller $d E / d x$ than do inelastic protons. The difference between the $d E / d x$ of a proton and that of a pion that stop in the same counter may be seen in Table VII. Even though all the other criteria imposed ought to select only inelastic protons, the pulse-height spectrum of events for each stopping counter was recorded. 


\section{Proton Data Analysis}

The proton-energy interval for each stopping counter was determined by the amount of material placed before that particular counter and the counter thickness. All protons had to traverse equal amounts of material in the telescope before entering a stopping counter, but depending on where the initial collision took place in the hydrogen target a variable thickness of hydrogen was traversed. In order to obtain the average-initialenergy interval for each stopping counter given in Table VII, an average of the distance traveled in hydrogen was used.

Elastic protons were used to check and calibrate the stopping channels. When the elastic-conjugate-pion counter was placed in coincidence instead of anticoincidence, the telescope counted only elastic protons. For each stopping channel, the elastic protons of the proper energy were about $10 \mathrm{deg}$ beyond the kinematic limit for inelastic protons of that energy [see Fig. 1(b)]. Since the elastic kinematics are known and the cross section is large, a convenient and abundant source of protons of variable energy was available for calibrating the telescope. A measurement of the yield of elastic protons in a particular channel of the telescope was taken as a function of angle, and the acceptance of a particular stopping channel was determined by using the elastic kinematics to convert from angle to energy. The calibrations agreed well with the energy intervals calculated by using the thickness of absorbers.

The solid angle was determined by a geometric calculation, which took into account the finite sizes and shapes of the beam and target.

As stated in Sec. IIIB1, only inelastic protons should appear in a $d E / d x$ analysis of events that were counted in the telescope. The $d E / d x$ counter was calibrated on elastic protons in order to select the minimum acceptable pulse height for each stopping channel. This criterion was set so that at least $99 \%$ of the calibration protons were accepted. The inelastic data were analyzed two ways: first, by using no minimum-pulse-height criterion; and second, by using the minimum-pulse-height criterion for protons, mentioned above. Within the statistical accuracy of the measurement, the two methods gave the same results for the yield. Thus, as stated above, the $d E / d x$ criterion, which was only a check, was not needed to identify inelastic-proton events. However, the results stated in this paper include the minimum-pulse-height criterion.

A number of corrections were considered and applied to the raw data before cross sections were extracted. A certain percentage of protons did not reach their stopping counter because of nuclear collisions. A knowledge of all the charged-reaction products and their energy and angular distribution as a function of proton energy would be necessary, in order to calculate reliably a correction for these collisions. There is not enough information on these processes to make a good

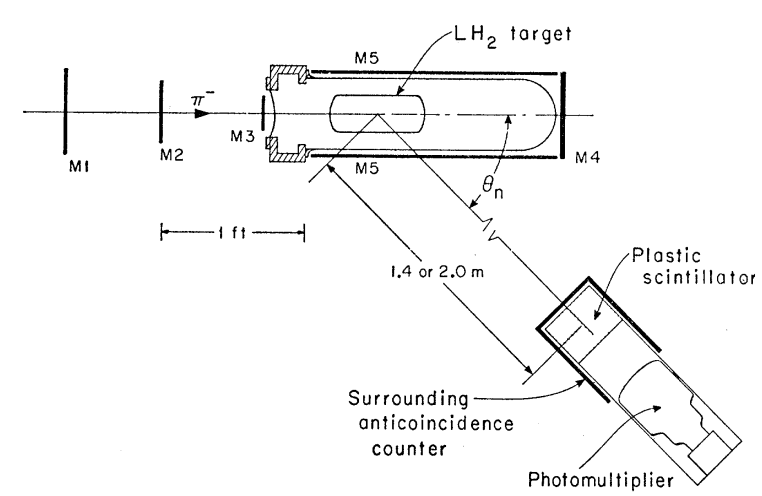

FIG. 11. Diagram of the inelastic-neutron-detection system. Simultaneous measurements were made with four neutron counters such as those shown. The liquid-hydrogen target was a horizontal cylinder 3 in. in diameter and 8 in. long.

calculation. Fortunately, a measurement of losses due to nuclear collisions for a telescope very similar to the one used in this experiment has been carried out by Cence. ${ }^{27}$ By use of the nuclear-collision losses measured by Cence, adjusted for the proton-energy intervals used in this experiment, corrections were made to the protonenergy spectra. These corrections varied from $3 \%$ in the lowest energy interval to $14 \%$ in the highest energy interval.

Multiple Coulomb scattering in the telescope was minimized by making each successive counter larger than the previous one. The calculation of Coulomb scattering was based on the geometric calculations of Sternheimer. ${ }^{26}$ It was assumed that the protons were uniformly distributed over the surface of $\mathrm{S}_{1}$. Also, the direction of the protons was assumed to be parallel to the axis of the stopping counters. The calculated correction was less than $2 \%$ for all counters.

Statistical fluctuations in the energy loss of protons in the scintillator and copper used in the telescope were calculated by following the method of Rossi. ${ }^{28}$ It was found that both the fluctuations in energy loss in the $d E / d x$ counter and the fluctuations in range were negligible.

\section{Experiment III-Measurements of the Neutron in the $\pi^{+} \pi^{-} n$ and $\pi^{0} \pi^{0} n$ Reactions}

\section{Inelastic-Neutron-Detection System}

The experimental arrangement is shown in Fig. 11. The incident- $\pi^{-}$flux was monitored by scintillation counters M1, M2, and M3. Neutral particles produced in interactions between the $\pi^{-}$beam and protons of the liquid hydrogen target were detected by observing the charged products of their interactions in plastic scintillator. Neutrons produced in inelastic $\pi^{-}-p$ interac-

${ }^{27}$ Robert J. Cence and Burton J. Moyer, Phys. Rev. 122, 1634 (1961).

${ }^{28}$ B. Rossi, High Energy Particles (Prentice-Hall, Inc., Englewood Cliffs, New Jersey, 1952), pp. 29-37. 
tions were identified by measuring the time-of-flight (i.e., velocity) distribution of the neutral particles. The time interval between the passage of a $\pi^{-}$through the beam-monitor counters and the detection of a neutral particle was measured electronically by time-to-height conversion (THC) and pulse-height analysis (PHA).

The neutral-particle detector was a 4 -in.-thick piece of plastic scintillator with a 40 -in. ${ }^{2}$ cross-section area viewed by a photomultiplier (Amperex 58AVP). This counter was surrounded by an anticoincidence counter to reject charged particles. The method of obtaining the timing information from the photomultiplier signals was pulse differentiation to produce a zero-crossing signal whose zero-crossing point was detected by a tunneldiode discriminator. ${ }^{29}$ The differentiated photomultiplier pulse provided a signal whose timing information was nearly independent of the scintillation-light amplitude in the expected 50:1 range of fluctuation. The efficiency of this detector was a function of the lowest light output the system could detect. This threshold for detection was calibrated with the Compton electron pulse-height spectrum of a $\mathrm{Na}^{22}$ gamma source. The detection threshold was set at a level corresponding to the light produced by a $2.3-\mathrm{MeV}$ electron.

In order to reduce the signal rate on the beam side of the time-of-flight system, counter M4, located after the hydrogen target, rejected incident $\pi^{-}$'s that were not scattered by an angle greater than $13 \mathrm{deg}$ in the hydrogen. The M5 was a cylindrical scintillation counter surrounding the hydrogen target used to distinguish neutral particles accompanied by charged particles from those not accompanied by charged particles.

The normal separation between the hydrogen target and the neutron detectors was $1.4 \mathrm{~m}$. The hydrogen target was not defined by the neutral-particle detectors. Presumably the target-full, target-empty subtraction should eliminate non-hydrogen-derived background. To check the validity of the measurement, a large amount of data, particularly at $374 \mathrm{MeV}$, was taken at a separation of $2.0 \mathrm{~m}$. This decreased the solid angle subtended at the target by a factor of 2 , and a corresponding decrease in yield should have occurred for target-derived particles.

The neutron detectors had a sizable probability (approx 15\%) of detecting photons by the processes of Compton scattering or pair production in the scintillator. Because of their single velocity, all photons from the target were located in one peak in the time-of-flight spectrum. The location of this peak in conjunction with the known conversion coefficient of the THC-PHA system provided an absolute time-scale calibration. The width of the photon peak was a resultant of the resolution of the time-of-flight analysis system and the fluctuations in flight path due to the finite size of the target and the detectors. The flight-path resolution

${ }^{29}$ Arthur E. Bjerke, Quentin A. Kerns, and Thomas A. Nunamaker, Lawrence Radiation Laboratory Report UCRL-9893, August 1961 (unpublished). function was computed as a function of detector angle. The measured photon peak widths indicated that the time-of-flight system resolution was $0.8 \mathrm{nsec}$, half-width at half-maximum.

\section{Neutron Data Analysis}

The raw data consisted of the neutral-particle yield from hydrogen as a function of time of flight. The objective of the data analysis was to extract the yield of neutrons from inelastic reactions and transform it to the differential cross sections as a function of neutron energy and angle, $d^{2} \sigma / d T d \Omega$. The time-of-flight spectrum was separated into the contributions of photons, neutrons from the reaction $\pi^{-} p \rightarrow \pi^{0} n$, and inelastic neutrons presumed to be from $\pi^{-} p \rightarrow \pi^{+} \pi^{-} n$ (charged mode) and $\pi^{0} \pi^{0} n$ (neutral mode).

Because the principal source of photons was the charge-exchange reaction, most of the photons appeared in the neutral-mode data. Photons from the reactions $\pi^{-} p \rightarrow \pi^{-} \pi^{0} p$ and $\pi^{-} p \rightarrow \pi^{-} \gamma p$ were located in the charged-mode data. The $\gamma$ peaks of the time-of-flight spectra were fitted by a Gaussian function (with the amplitude, center, and variance as parameters) and subtracted from the neutral-particle yield. In all cases the $\gamma$ contribution was clearly resolved.

The charge-exchange neutrons were included in the neutral-mode data. The separation of the neutrons from charge-exchange and inelastic reactions was much more ambiguous than the $\gamma$ situation. At a given detector angle the charge-exchange neutron energy was unique and consequently these neutrons were located in a peak. However, the over-all resolution in measurements of the neutron spectra was more complicated than the problem in the $\gamma$ case. In addition to the time-of-flight system resolution, the following factors had to be considered: (a) the finite angular resolution of the neutron detectors; (b) the flight-path uncertainty; and (c) the $\pi^{-}$-beam-energy spread. The spread of charge-exchange neutron energy and the inelastic kinematic limit resulting from the finite angular resolution can be visualized by considering the kinematics [see Fig. 1(b)].

For data at angles $>45 \mathrm{deg}$, the charge-exchange neutrons dominated the spectra and were unambiguously subtracted. The shape of the charge-exchange peak agreed in all cases with the calculated resolution. For data at angles $<45 \mathrm{deg}$ the separation in time between the charge-exchange neutrons and the maximumenergy inelastic neutrons was $\approx$ or $<$ the total resolution. The charge-exchange contribution was subtracted by normalizing the area of the calculated resolution function to twice the observed yield between the center of the charge-exchange peak and a point two standard deviations from the center in the direction away from the inelastic portion of the spectrum.

The results were corrected for three types of effects: (a) over-all normalization adjustments, (b) conversion of photons leaving the hydrogen target, and (c) multiple 
scattering of neutrons. The beam anticoincidence counter necessitated a correction for the number of charged-mode events lost because one of the charged pions accompanying the neutron traversed M4. The fraction lost was estimated to be $5 \%$ from the $\pi^{+}$angular distributions.

A fraction of the all-neutral final-state reactions did not appear in the neutral-mode data because: (a) a $\gamma$ interacted with material in the target or surrounding counters and produced an $e^{+}, e^{-}$pair, or (b) a $\pi^{0}$ decayed in the $\gamma+e^{+}+e^{-}$mode. If one of the resulting charged particles passed through M4, the event was lost. If one passed through M5, the event was recorded in the charged-mode data. The probability of conversion averaged over $\gamma$ energy and direction of escape was computed for $\gamma$ 's resulting from both charge-exchange and inelastic $\pi^{0}$ 's. These probabilities and the known branching ratio for internal-conversion mode of $\pi^{0}$ decay $(1.2 \%)$ were used to make the appropriate adjustments to the data. The fraction of events lost was $<1 \%$ and the fraction incorrectly identified was $10 \%$. To check this calculation, some data were taken with 0.02 in. of $\mathrm{Cu}$ between the target and M5 to increase the $\gamma$ conversion. These measurements agreed with the expected results.

The rescattering of neutrons in the hydrogen or surrounding materials affected the data in two ways. The energy lost by a charge-exchange neutron in rescattering could result in a final neutron energy in the kinematically allowed range for an inelastic reaction. The most significant succession of processes, $\pi^{-}+p \rightarrow \pi^{0}+n$ followed by $n+p \rightarrow n+p$, was analyzed in detail. The differential distribution in energy and angle of the rescattered neutrons was computed and subtracted from the inelastic data. The over-all correction was small $(<7 \%)$, but because the calculated energy distribution was strongly peaked at low neutron energies it was as high as $50 \%$ in the region of $10 \mathrm{MeV}$. The rescattering of inelastic neutrons could distort the differential distributions in energy and angle. The over-all effect was estimated to be small $(\approx 5 \%)$, but as in the chargeexchange case the effect would be most significant at low neutron energies. No adjustment was made to the data for this effect. Therefore the differential distributions for neutron energies below $50 \mathrm{MeV}$ should be regarded with reservations that increase as the energy decreases.

The neutron-detection efficiency of plastic scintillator is a function of the light-detection threshold and the detector geometry. For this analysis the efficiency was computed by using all known systematics for neutroncarbon interactions and neutron-hydrogen interactions. The calculation included first and second scattering contributions from both hydrogen and carbon interactions, saturation effects in the production of scintillation light, and finite resolution of the detection threshold. Reference 30 contains a complete description of the efficiency calculation used in the present data analysis.

\section{ACKNOWLEDGMENTS}

We would like to acknowledge the help, support, and encouragement received from Professor A. C. Helmholz and Professor B. J. Moyer throughout the course of this work. The participation of Dr. Paul G. McManigal in the early phases and the assistance of Howard Goldberg in setting up the experiments and taking data are greatly appreciated.

Finally we would like to thank James Vale and the members of the cyclotron crew for their help and cooperation during these experiments.

${ }^{30}$ Richard J. Kurz, Lawrence Radiation Laboratory Report UCRL-11339, March 1964 (unpublished). 